Journal of Al-Azhar University Engineering Sector

Vol.16, No. 59, April 2021, 442-462

\title{
EGYPTIAN GOVERNMENTAL UNIVERSITIES "AN INDUCTIVE STUDY ON THE ORIGIN AND REALITY OF EGYPTIAN GOVERNMENTAL UNIVERSITIES"
}

\author{
Shaban Taha Ibrahim¹, Tarek Saad Alhenawy ${ }^{1}$, Ahmed Elsayed Rashiedy ${ }^{* 1}$ \\ ${ }^{1}$ Department of architecture, Shoubra faculty of engineering - Benha university, Qalyubiyah, Egypt. \\ *Corresponding Author E-mail: Ahmed.Ibrahim@feng.bu.edu.eg
}

\begin{abstract}
In its inception, Egyptian governmental universities went through many stages that affected their current reality, although this inception was affected by economic, social, and political situation, but it established a reality in which many of the strengths and challenges facing Egyptian governmental universities appear. These challenges represent the basis that must be reviewed and studied to develop a comprehensive vision for the development of governmental universities. The study aims to identify the challenges facing Egyptian governmental universities, thus contributing to develop appropriate futuristic plans to deal with those challenges, without neglecting the strengths of the higher education system and the Egyptian governmental universities. based on an inductive approach in reviewing the inception of Egyptian governmental universities and the impact of this inception on the challenges they face now or in the future.

The study concluded the affection of Governmental universities' inception on its current situation because of the absence of long-term plans to deal with educational developments, or developments in the political, economic, and social nature of Egyptian society, Emphasizing the need for plans and objectives to develop Egyptian governmental universities include mechanisms to deal with all of their challenges, as these challenges represent an interlocking system that affects each other.

The study recommended to establish a comprehensive vision for the development of Egyptian governmental universities that seeks to provide an attractive educational environment capable of keeping pace with the developments that occur at the educational, political, economic, and social levels. Reviewing the mechanisms for selecting governmental universities' sites, relevance to admissions' numbers and coverage areas for each campus, to help limiting several problems, including expatriation. Finally, Developing Egyptian governmental universities' campus design mechanisms and criteria to keep pace with the various developments of universities' design around the world without neglecting our Egyptian reality.
\end{abstract}

KEYWORDS: University, Egyptian governmental universities, University campus, Educational process, Higher Education. 


\title{
الجامعات الحكومية المصرية
}

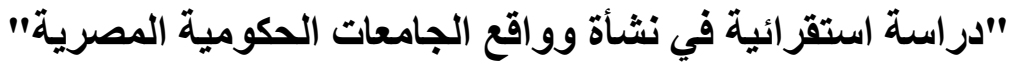

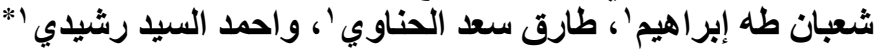

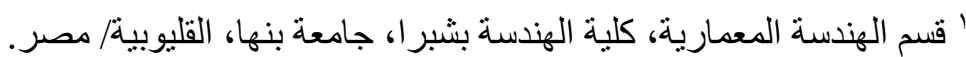

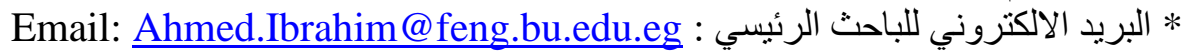

الملخص:

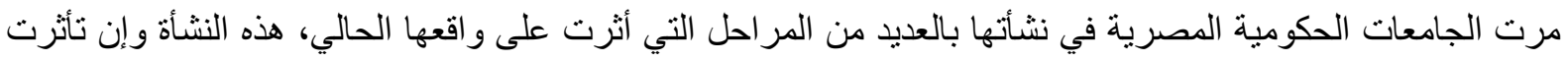

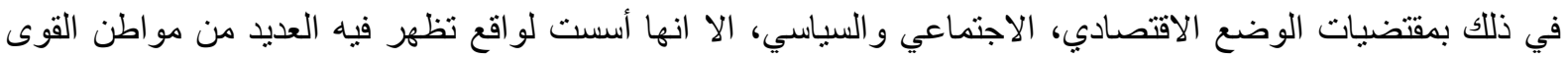
و التحديات، والتي تتقسم الى تحديات الطبيعة والاسس التصميمية للحرم، تحديات إدارية تشغيلية وأخير ا تحديات تعليمية.

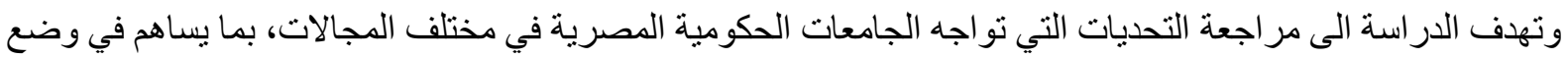

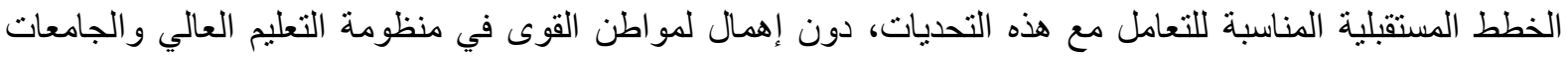

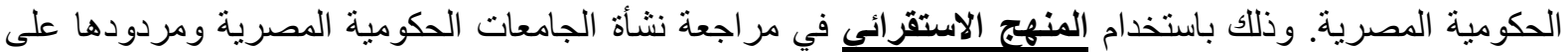
التحديات التي تو اجهها حالياً او مستقبلياً وخلُصت الدر اسة الى بيان أثر مر احل نشأة الجامعات الحكومية على وضعها الحالي نتيجة لغياب الخطط طويلة الاجل

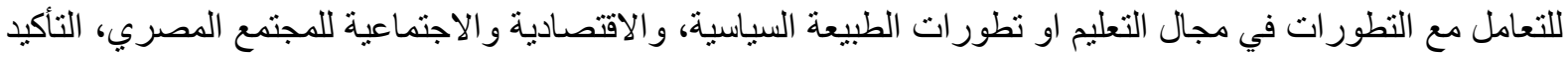

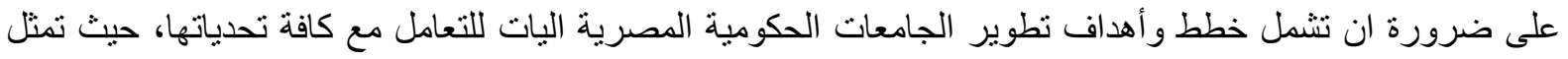
هذه التحديات منظومة منشابكة يتأثر كلٍ منها بالآخر. واوصت الدر اسة بالعمل عل تأسيس رؤية شاملة لتطوير الجامعات

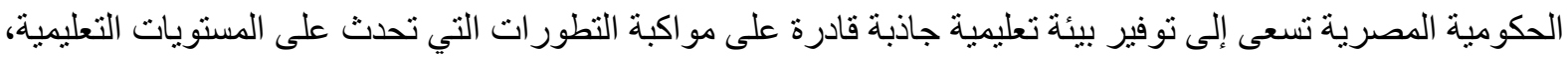

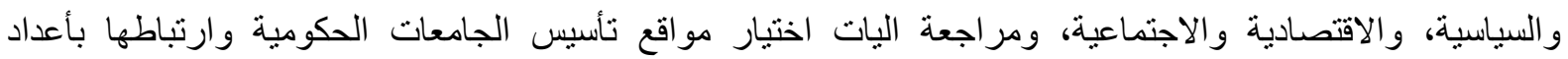

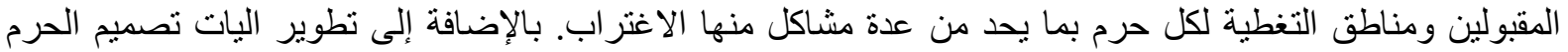

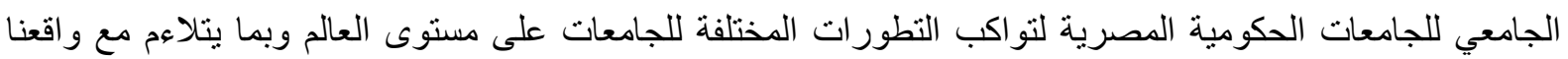
المصري.

• الكلمات المفتاحية: الجامعة، الجامعات الحكومية المصرية، الحرم الجامعي، العملية التعليمية، التعليم العالي.

1 المقدمة:

تعرف الجامعة بأنها المؤسسة التربوية العلمية المنظمة التي تقع على قمة السلم التعليمي في المجتمع وتقوم بأعداد الفرد

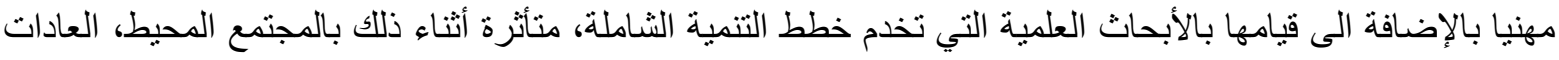

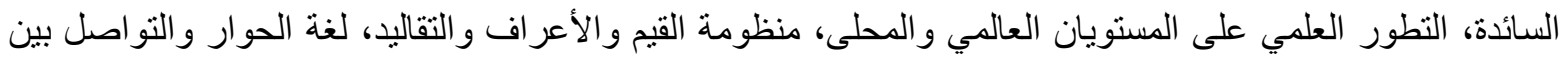

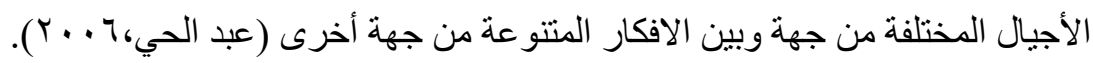

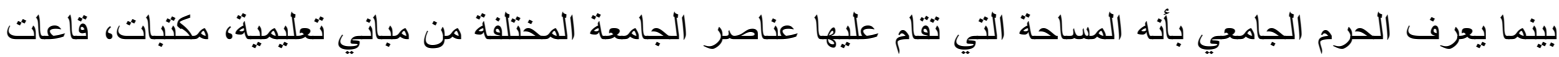

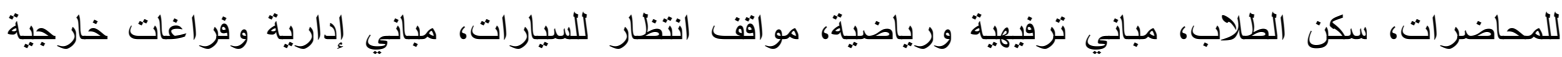

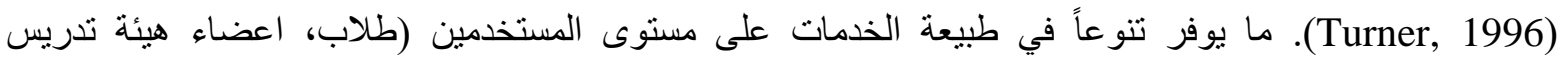

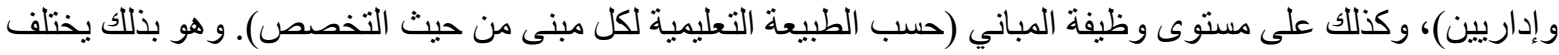
عن مفهوم الحرم الخاص بكلية واحدة فقط والذي قد لا يحتوي على بعض عناصر حرم الجامعة مثل سكن الطلاب و المباني الرياضية. يوضح كلا التعريفين الارتباط و التأثر بين ما تتعرض له الجامعة من قضايا وبين الحالة التي يكون عليها الحرم الجامعي.

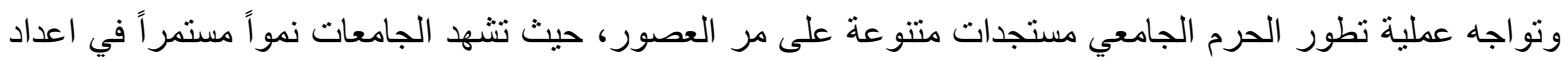
الطلاب واعضاء هيئة التدريس، زيادة استخدام وامتلاك السيارة للفرد الواحد (الامر الذي يمثل قضية هامة للحرم

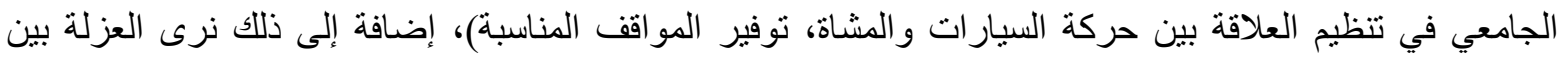


الجامعات الحكومية المصرية "در اسة استقر ائية في نشأة وو اقع الجامعات الحكومية المصرية"

الجامعة والمناطق المحبطة، الفصل بين سكن الطلاب و الجامعة، التحو لات و التطور المستمر في ممارسات التعليم وسوق التعليم العالمي و غير ها من التحديات التي ناقتشها العديد من الدر اسات (Mitchell \& Vest,2007).

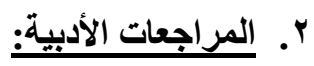

يرجع استخدام مصطلح الجامعة (الكلمة اللاتينية الأصل "universitas") لأول مرة في وقت تجدد الاهتمام بالتقاليد

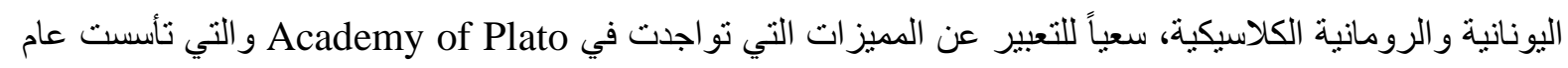

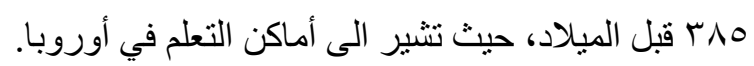

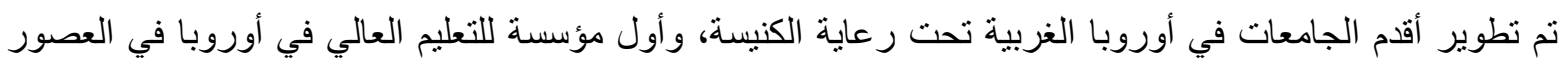
الوسطى كانت جامعة القسطنطينية في تركيا، تليها جامعة سال يرنو (القرن التاسع) في إيطاليا حيث كانت دئ ديرًا

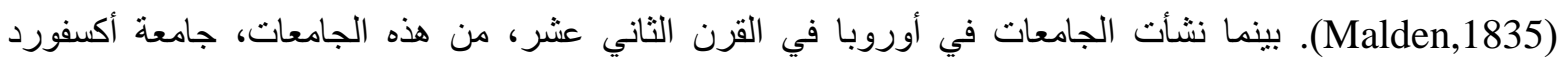

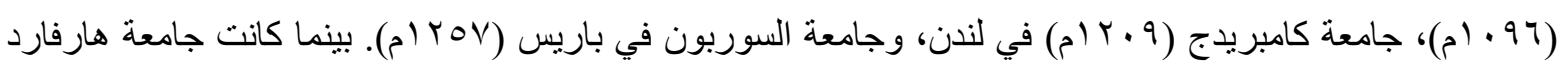

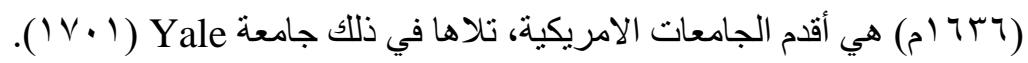

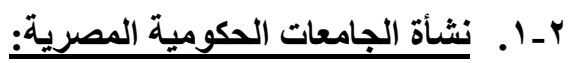

يستطيع الباحث في تاريخ التعليم المصري ان يحدد بيساطه نقطة البداية في ناريخ نشأة الجامعات في مصر ، حيث يظهر

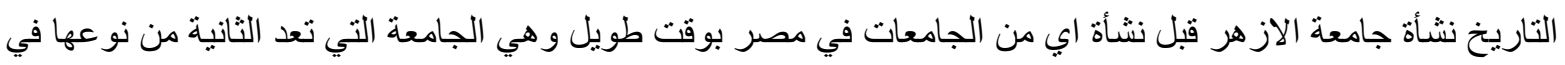

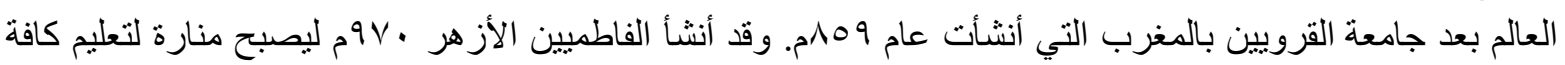

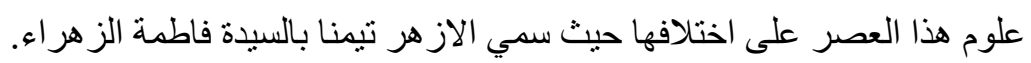

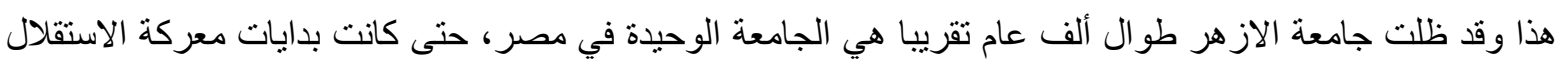

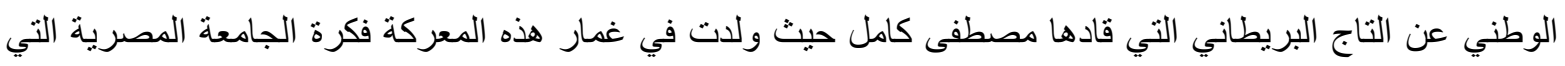

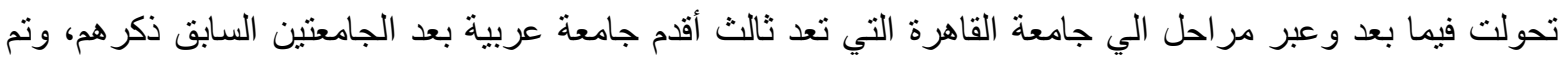

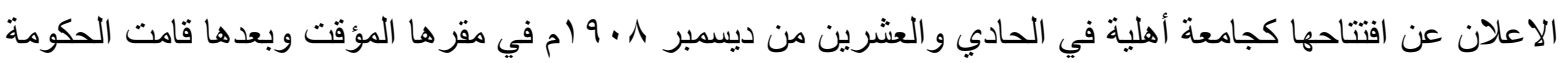

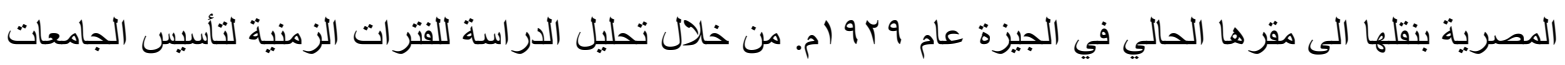

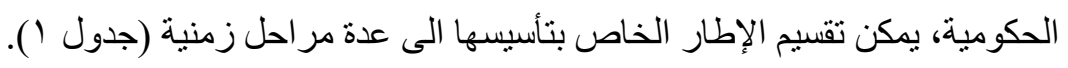

\section{r. مواطن القوة في منظومة التعليم والجامعات الحكومية المصرية:}

من خلال الرؤية الواردة بتوصيات المؤتمر القومي للتعليم العالي تم تحديد العديد من مواطن القوة في منظومة التعليم

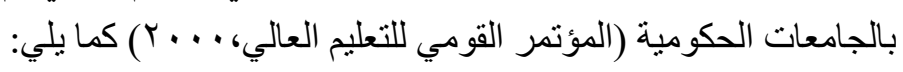

$$
\text { تز ايد الطلب المجتمعي على خدمات التعليم العالي. }
$$

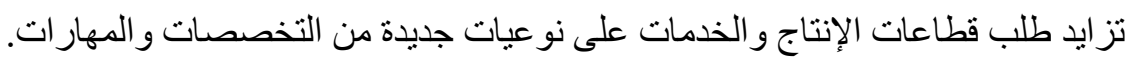
توفر فرص التوسع في التعليم بالخروج الى مناطق جديدة وتقديم نماذج منطورة لمؤسسات التئ التعليم العالي أكثر ارتباطا وتفاعلا بالبيئة. وجود هيكل ضخم من أعضاء هيئة التدريس يمكن استثماره بصورة فعالة في عملية تطوير الجامعات و العملية التعليمية. وجود قاعدة طلابية عريضة تمتلك قدرات تجعلها أقدر على استيعاب التطور المتوقع في الأداء العلمي بمنظومة التعليم العالي. وجود هيكل من الإمكانات المادية المتمثلة في المباني و المو اقع التي تشغلها الجامعات يمكن تطوير ها بصورة ترفع من كفاءة المنظومة التعليمية. وجود طلب متز ايد وسوق أكثر استعدادا لاستيعاب المزيد من مخرجات المنظومة التعليمية. 
الجامعات الحكومية المصرية "در اسة استقر ائية في نشأة وو اقع الجامعات الحكومية المصرية"

جدول ا ـ مر احل نشأة الجامعات الحكومية المصرية

\begin{tabular}{|c|c|c|c|c|}
\hline \multicolumn{2}{|c|}{ الجامعات التي تم تأسيسها } & السمات الحاكمة لهذه المرحلة & الفترة الزمنية & المرحلة \\
\hline $19 \cdot 1$ & - - جامعة القاهرة & \multirow{4}{*}{ 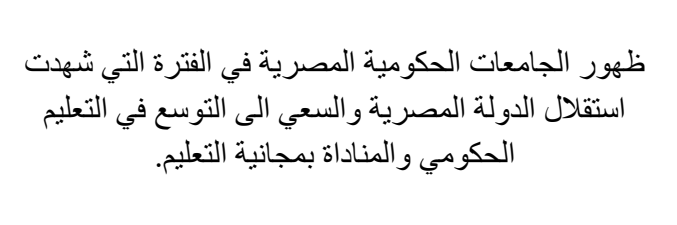 } & \multirow{4}{*}{$\begin{array}{l}\text { م } 190 \mathrm{~V} \\
\text { متى } 19.1\end{array}$} & \multirow{4}{*}{ التأسيس } \\
\hline $19 \leqslant Y$ & - جامعة الإسكندرية & & & \\
\hline 190. & - - جامعة عين شمس & & & \\
\hline $190 \mathrm{~V}$ & - جامعة أسيوط & & & \\
\hline $19 V Y$ & - جامعة طنطا & \multirow{7}{*}{ 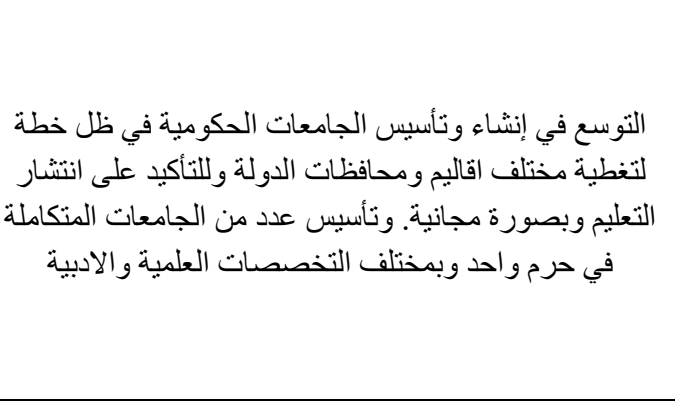 } & \multirow{7}{*}{$\begin{array}{l}\text { pl9V7 } \\
\text { متى } 9 \vee r\end{array}$} & \multirow{7}{*}{ الأنتشار } \\
\hline $19 V Y$ & - جامعة المنصورة & & & \\
\hline $19 \vee \leq$ & - - جامعة الزقازيق & & & \\
\hline $19 \times 0$ & - جامعة حلوان & & & \\
\hline $19 \times 7$ & - جامعة المنيا & & & \\
\hline $19 \times 7$ & - جامعة المنوفية & & & \\
\hline $19 \times 7$ & - جامعة قناة السويس & & & \\
\hline $199 \varepsilon$ & - جامعة جنوب الو ادي & 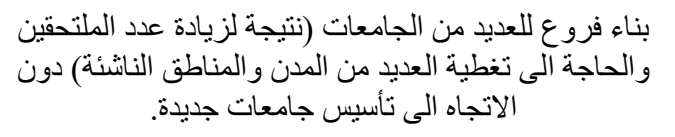 & \multirow{2}{*}{ 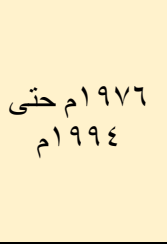 } & \multirow{2}{*}{ مرحلة ظهور } \\
\hline \multicolumn{3}{|c|}{ أدى ذلك الى عشو ائية المباني والحرم الجامعي للجامعات الحكومية المصرية، مع غياب النظرة المستقبلية الوضانها. } & & \\
\hline$r \ldots$ & - جامعة بني سويف & \multirow{6}{*}{ 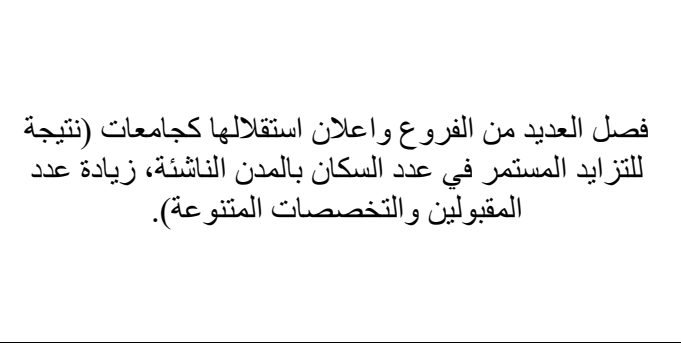 } & \multirow{7}{*}{ 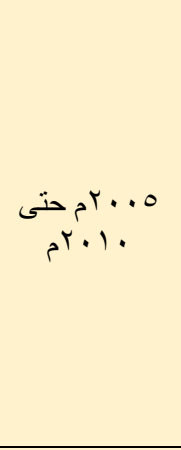 } & \multirow{7}{*}{ 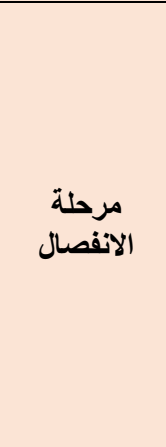 } \\
\hline r... & - - جامعة الفيوم & & & \\
\hline$r \ldots o$ & - جامعة بنها & & & \\
\hline$r \ldots r$ & - جامعة كفر الثيخ & & & \\
\hline$r \ldots r$ & - جامعة سو هاج & & & \\
\hline$r \ldots r$ & - جامعة العريش & & & \\
\hline \multicolumn{3}{|c|}{ 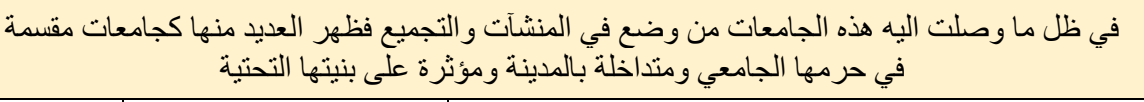 } & & \\
\hline$r \cdot 1 \cdot$ & - - جامعة بورسعيد & \multirow{6}{*}{ 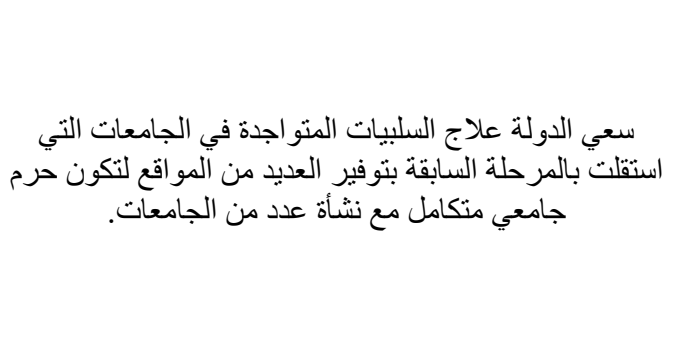 } & \multirow{7}{*}{ 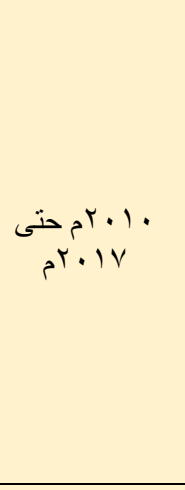 } & \multirow{7}{*}{ 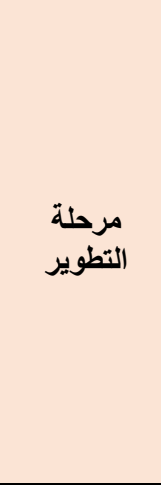 } \\
\hline$r \cdot 1 \cdot$ & - جامعة دمنهور & & & \\
\hline$r \cdot 1 r$ & - جامعة اسوان & & & \\
\hline r.Ir & - جامعة دمياط & & & \\
\hline$r \cdot 1 r$ & - جامعة السويس & & & \\
\hline$r \cdot 1 r$ & - - جامعة السادات & & & \\
\hline المقسمة & 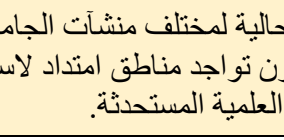 & 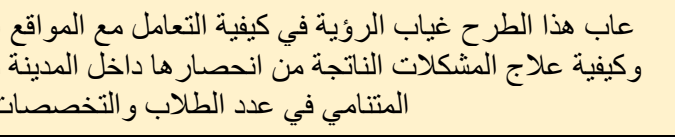 & & \\
\hline$r \cdot 11$ & - جامعة الو ادي الجديد & \multirow{3}{*}{ سعي الدولة الى تطبيق خطة انتشار الجامعات بتطبيق خطة القطاعين الحكومي } & \multirow{3}{*}{ 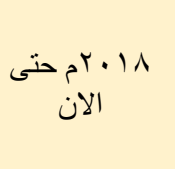 } & \multirow{3}{*}{ الانتثار } \\
\hline$r \cdot 11$ & - جامعة مطروح & & & \\
\hline$r .19$ & - جامعة الاقصر & & & \\
\hline
\end{tabular}

\section{؛. تحديات استخدامات الحرم الجامعى للجامعات الحكومية المصرية:}


الجامعات الحكومية المصرية "در اسة استقر ائية في نشأة وو اقع الجامعات الحكومية المصرية"

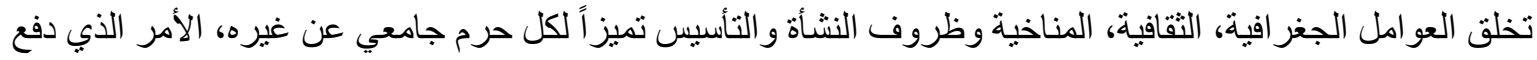

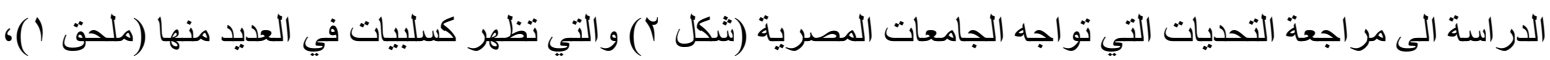

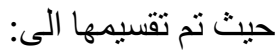

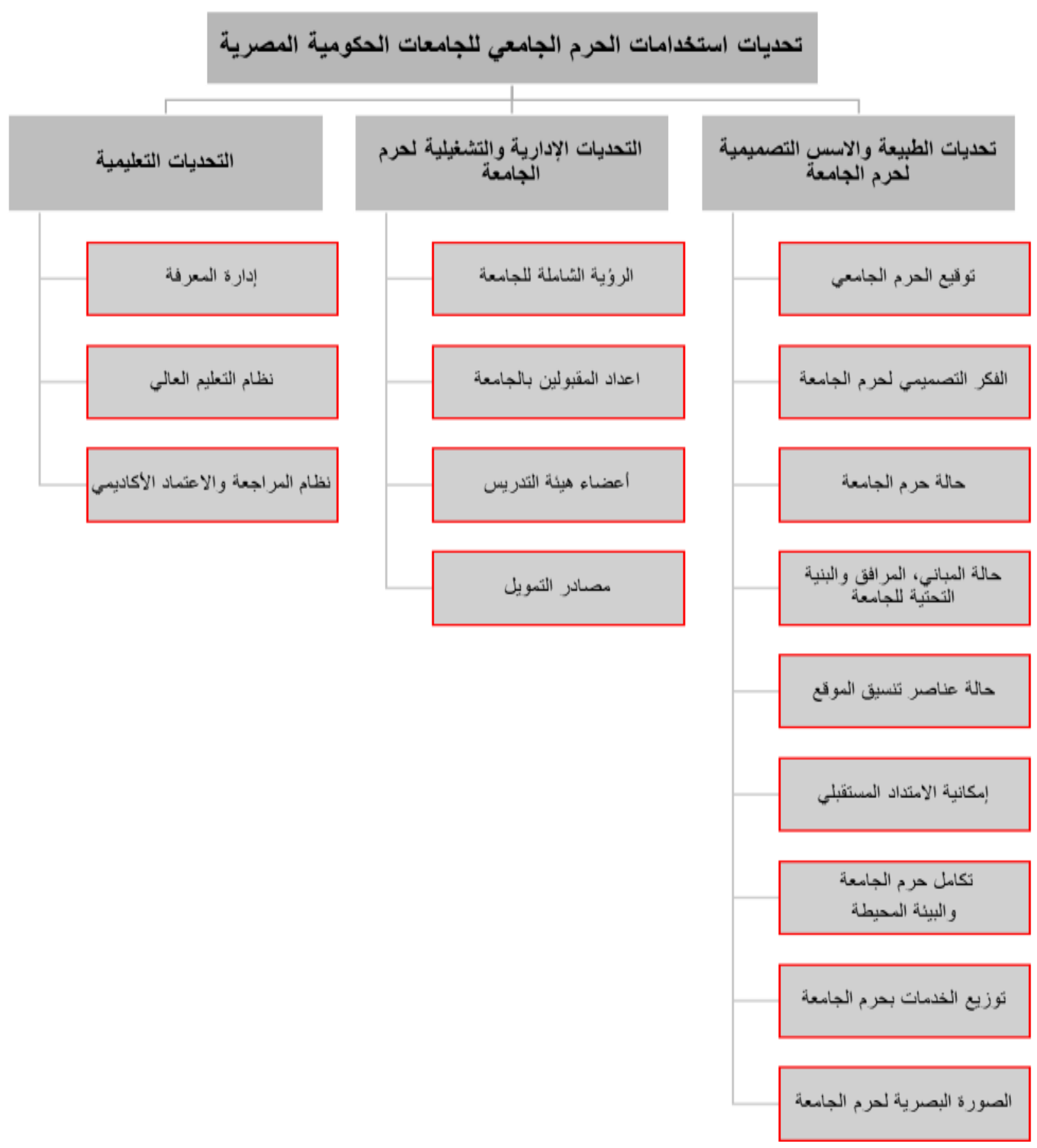

شكل ا ـ تحديات استخدامات الحرم الجامعي للجامعات الحكومية المصرية

ع ـ ا. تحديات الطبيعة والاسس التصميمية للحرم: و الخاصة بالمرجعيات و الاسس التي تقوم عليها الجامعة وحرمها

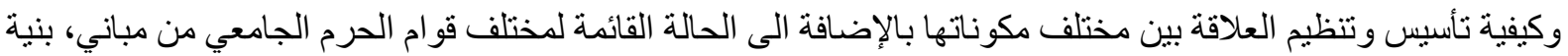
تحتية، مر افق و عناصر تنسيق الموقع.

ع ـ ا ـ ـ ت توقيع الحرم الجامعى: يعتبر اختيار موقع الجامعة من المؤثرات الهامة في عملية تخطيطها وتصميمها، حيث يمكن رصد العديد من التحديات المتعلقة بموقع الجامعة وو اقعها بالجامعات الحكومية المصرية كما يلي: 
- تأثير الجامعة على الواقع الاجتماعي، الاقتصادي للبيئة المحيطة: حيث يتبع تأسيس الجامعة تطور على المستوى الاجتماعي نتيجة لتعدد المستخدمين وما يتبعه من ثراء فكري نابع من هذه التعددية بما يؤثر على الطبيعة الاجتماعية للبيئة

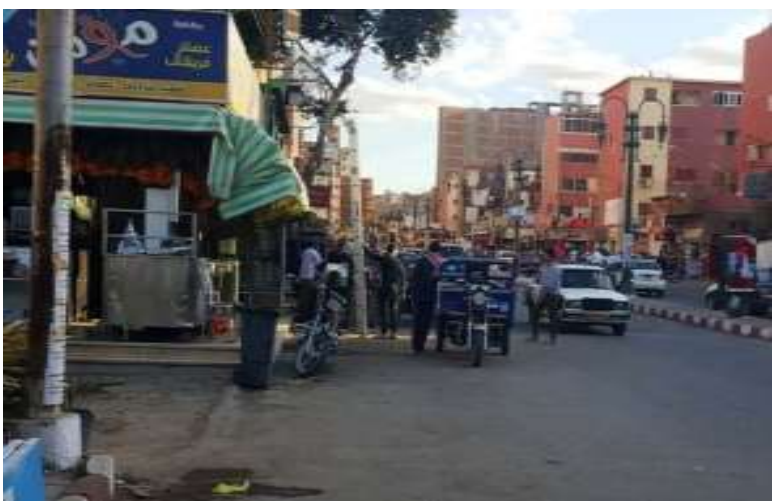

شارع المكتبات المتاخم لجامعة أسيوط

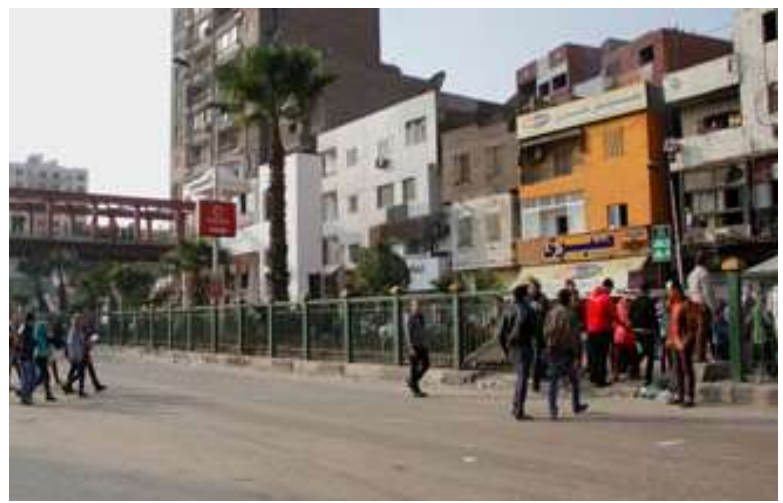

منطقة بين السر ايات المتاخمة لجامعة القاهرة

شكل r ـ ـ ظهور مناطق خدمات متنوعة متاخمة للجامعات

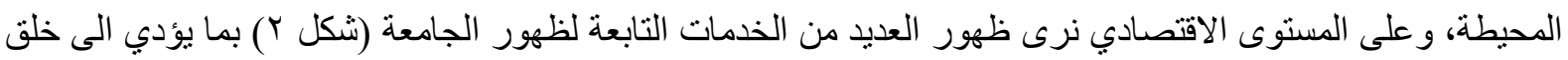
مزيد من فرص العمل في هذه الخدمات وتحسن في الو اقع لاقتصادي للمناطق المحيطة بالجامعة.

- أثثير الجامعة على خدمات البيئة المحيطة: يتبع تأسيس الجامعة في موقع ما نموا على مستوى مختلف الخدمات

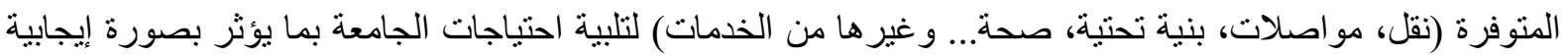

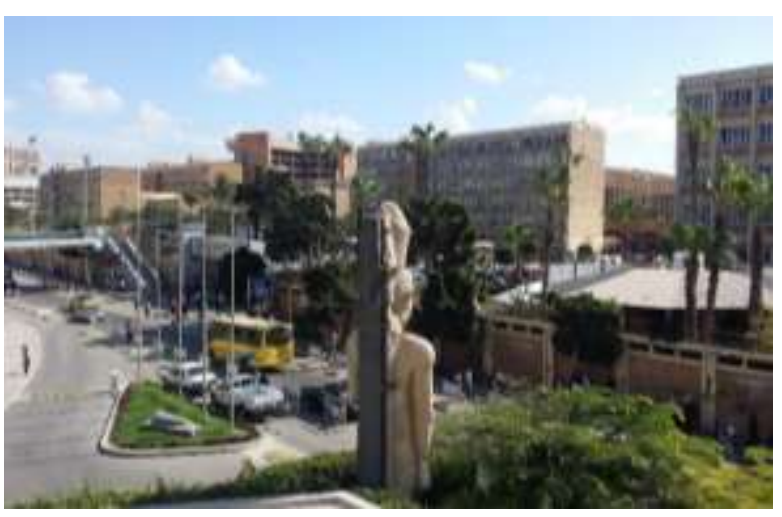

مدخل جامعة الاسكندرية

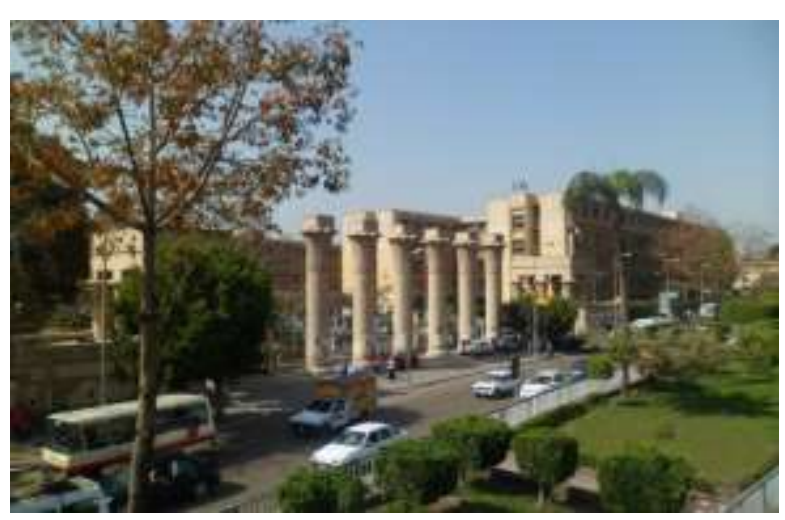

مدخل جامعة عين شمس

\section{شكل r ـ ت تأثير الجامعة على شبكة الطرق المحيطة}

على كافة المناطق المحيطة بها (شكل r). وهو ما دفع العديد من الدراسات (Anderson, 2010) الى الدعوة بإقليمية الجامعة بتأسيسها على أطر اف المدينة او خارجها والتأكيد على كونها عنصر جذب وتنمية للمناطق و المدن النانشئة.

ـ ـ الاغتراب: عند اختيار موقع الجامعة يجب ضرورة مر اعاة تأثثره على مسافات الحركة من والى الجامعة في ضوء

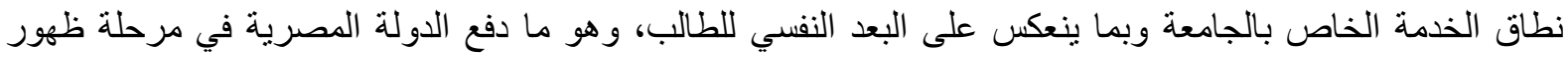

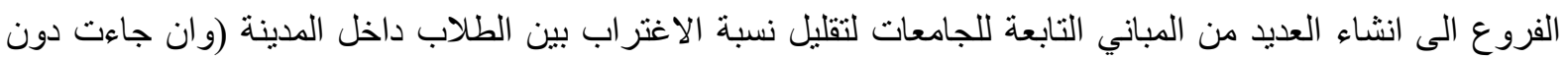
خطة مستقبلية لتأثير كثافات الاستخدام على المدينة).

- تأثير الجامعة على البنية التحتية للبيئة المحيطة: يمثل عدد المترددين على الجامعة من طلاب، أعضاء هيئة تدريس،

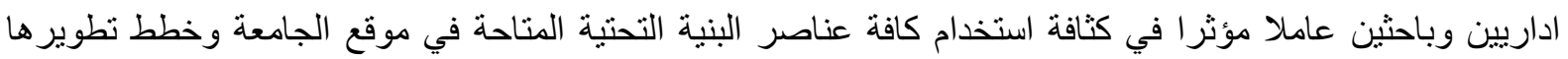

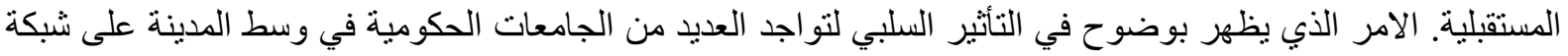
المو اصلات، الطرق، البنية التحتية و الخدمات الصحية. فنرى الازدحام في فترات الذروة، سو و وتهاللك البنية التحتية، سوء 
حالة شبكة الطرق والمواصلات... وغيرها من السلبيات الناتجة من كثافة الاستخدام حتى مع المحاو لات المستمرة من الدولة في علاج هذه السلبيات.

ع ـ ـ Y. الفكر التصميمى لحرم الجامعة: يشهد تصميم الجامعات تطور ا كبير ا بمرور الزمن نتيجة للتطور في الأساليب

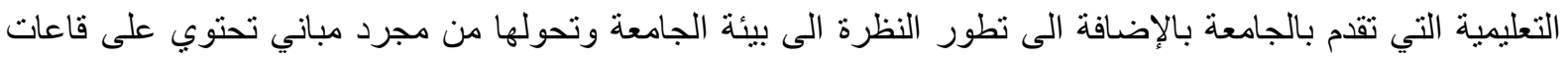
التدريس العديد من العلوم الى حديقة علوم تحتضن مختلف الأنشطة التعليمية، الاجتماعية والثقافية (Pearce, 2001).

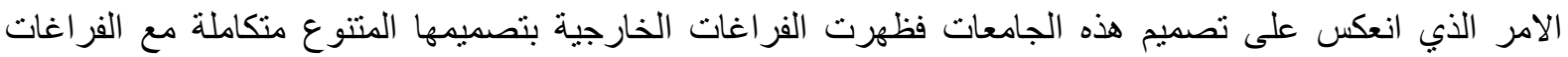
الداخلية للمباني في منظومة مركبة تهدف الى دعم العملية التعليمية المجتمعية التي تتم داخل الحرم الجامعي. - جمود الحالة التصميمة: حيث نرى مخططات الجامعة المصرية مرتبطة بالمباني دون الفر اغات الخارجية (شكل ع) )، حتى الجامعات التي تم تصميمها وفقا لرؤية تعتمد على التكامل بين مباني الجامعة وفر اغاتها الخارجية (جامعات حلون،

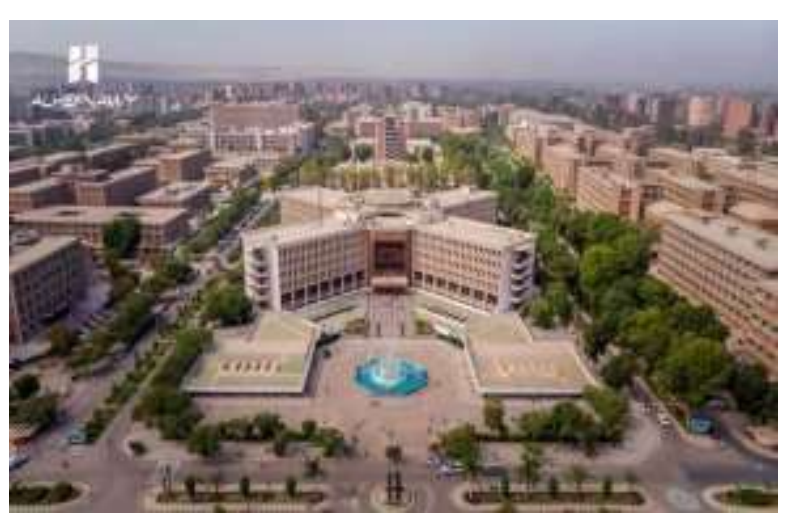

جامعة اسيوط

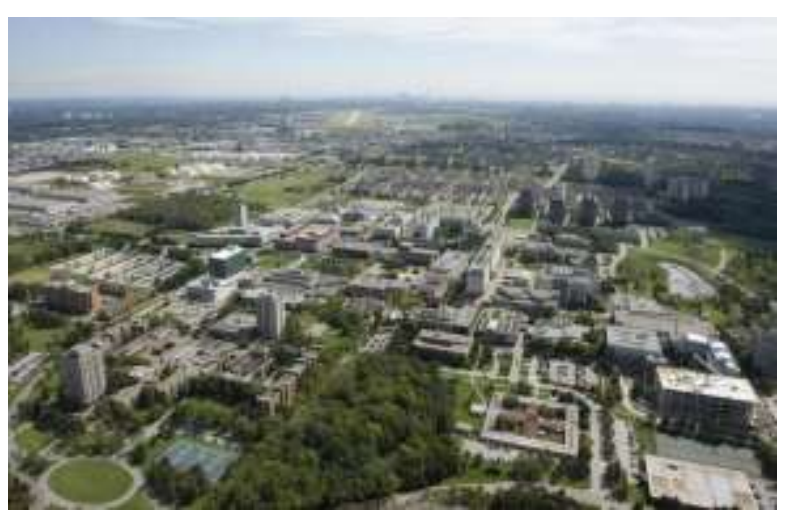

Toronto York University Campus

شكل ؛ ـ الاختلاف في التعامل مع مخطط الجامعة (بين الجامعة الغربية والجامعة المصرية)

أسيوط و المنيا) فقد تم تحويل هذه الفراغات الى طرق ومواقف لانتظار السيارات دون الالتفات الى دور ها الأساسي في دعم العملية التعليمية الاجتماعية للمستخدم.

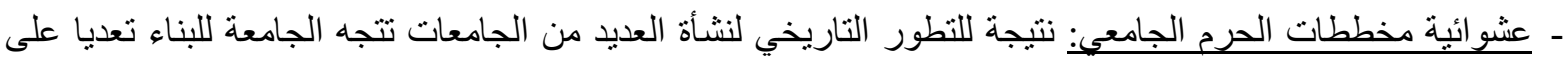

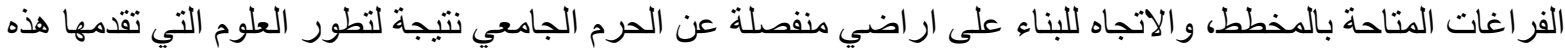

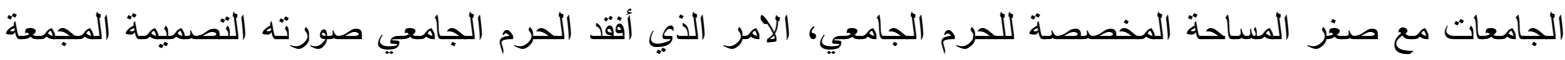
لمختلف العناصر، و الأنشطة التعليمية، و الترفيهية، و المجتمعية. ـ ـ ا ـ" ـ حالة حرم الجامعة: نتيجة لما سبق من تحديات ترتبط بتطور تأسيس الجامعات الحكومية، و الاعتماد على إنشاء

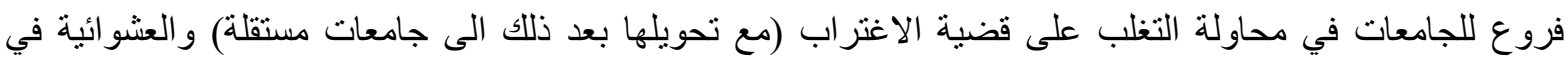
تصميم، تخطيط ونطوير هذه الجامعات، ظهر الحرم الجامعي للجامعات المصرية بصورة غلب عليها التقسيم والتشتيت،

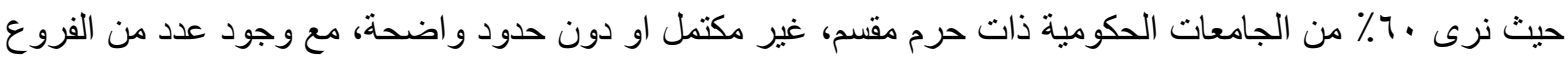

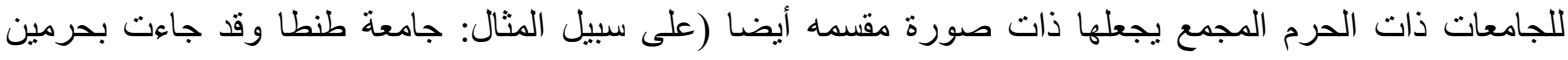

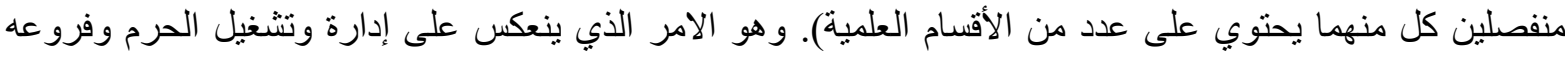

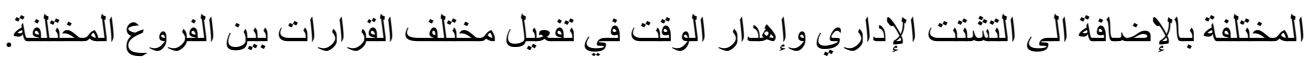

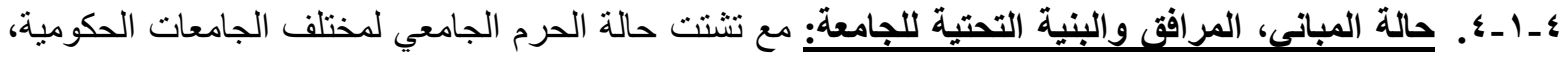

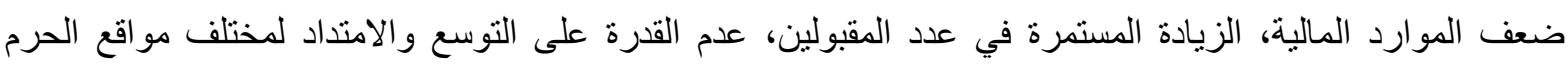

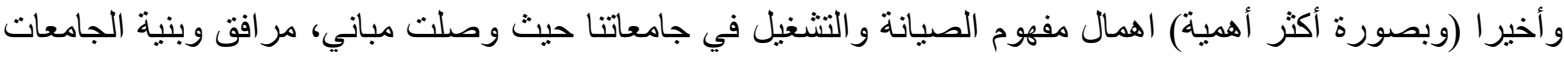

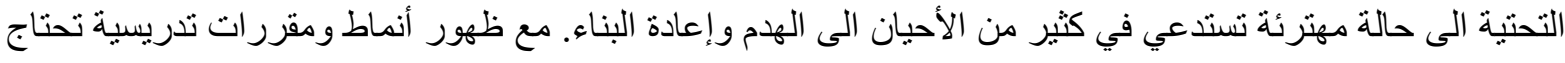

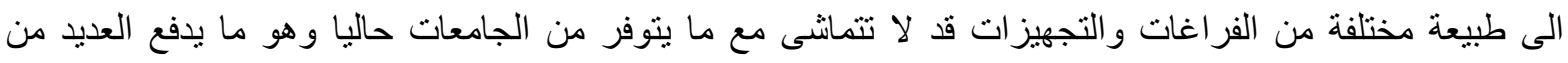




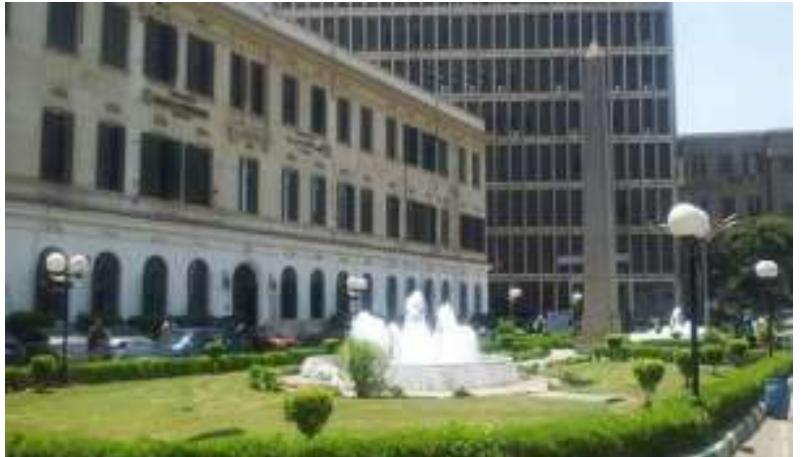

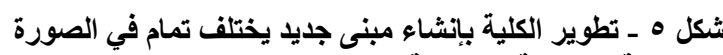

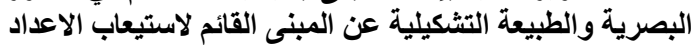

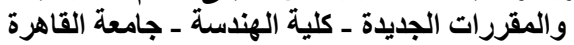

الجامعات الى تشييد مباني جديدة (شكل 0) تستوعب هذه الأنماط و المقررات وتز ايد اهمال المباني و المر افق القائمة

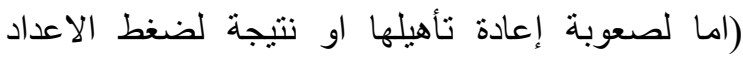

المتز ايد).

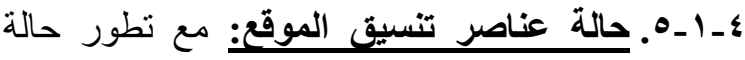

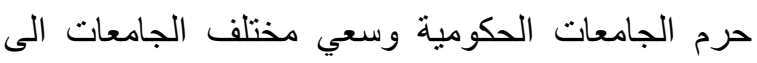
البناء لتلبية الاحتياجات من تزايد في اعداد المقيدين

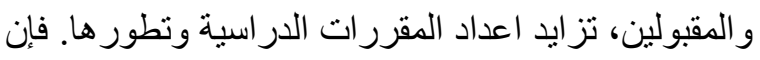

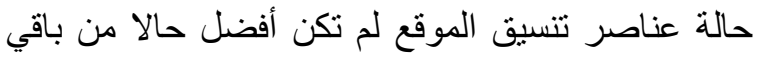
مرافق الجامعة حيث أصابها الإهمال في الصيانة

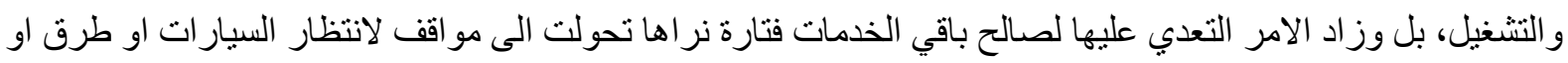

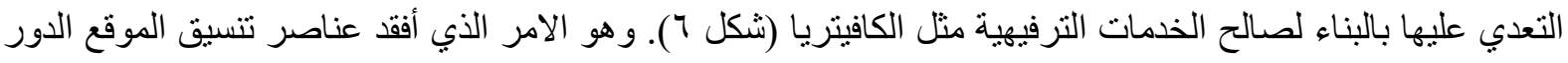

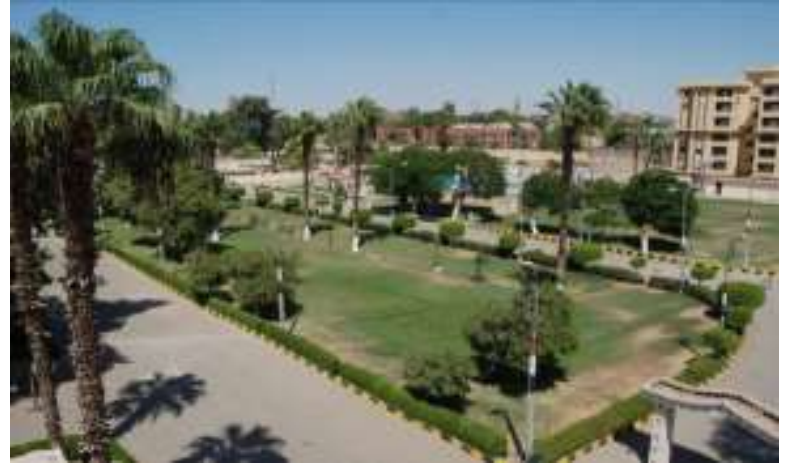

حالة الفر اغات الخارجية في جامعة اسيوط

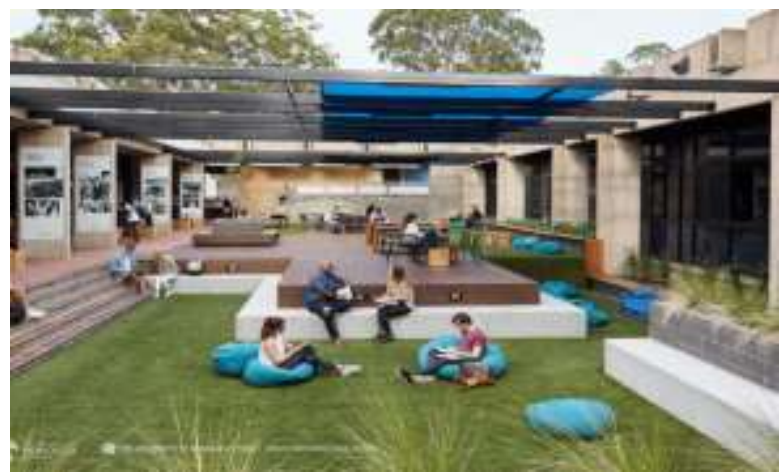

الفر اغات الخارجية في الجامعات الغربية

شكل 1 ـ الاختلاف في التعامل مع عناصر تنسيق الموقع والفراغات الخارجية بالجامعة الغربية والمصرية

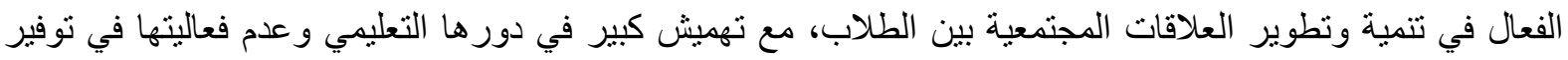

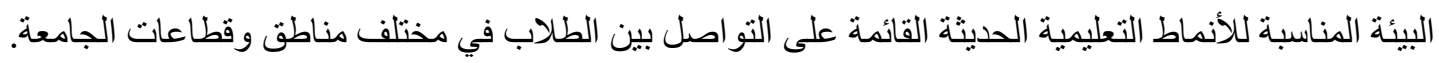

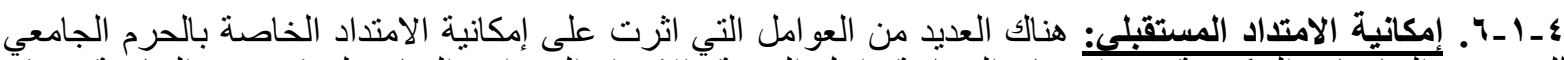

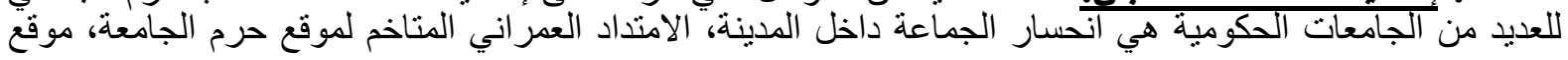

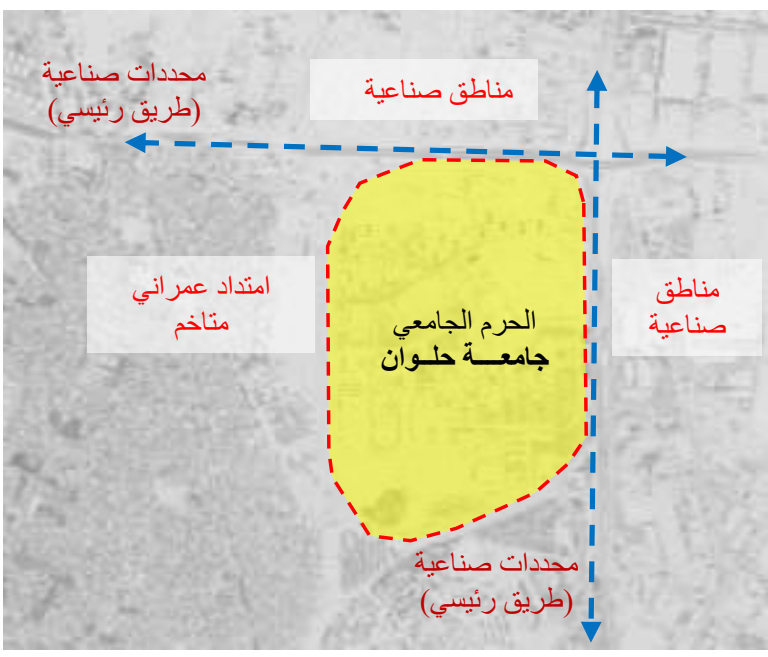

محددات الامتداد المستقبلي - جامعة حلــــــان

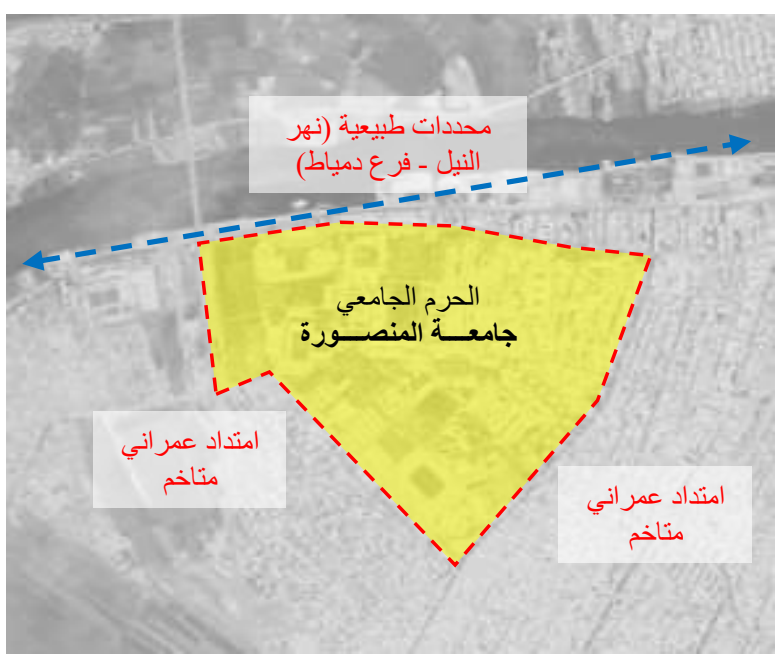

محددات الامتداد المستقبلي - جامعة المنصورة

شكل V - محددات الامتداد المستقبلي في حالات من الجامعات الحكومية المصرية 
الجامعات الحكومية المصرية "در اسة استقر ائية في نشأة وو اقع الجامعات الحكومية المصرية"

الجامعة و علاقته بالحدود الطبيعية والصناعية المحيطة، تو افر أر اضي غير صالحة للامتداد (شكل V).

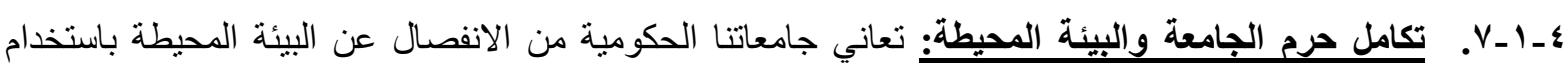

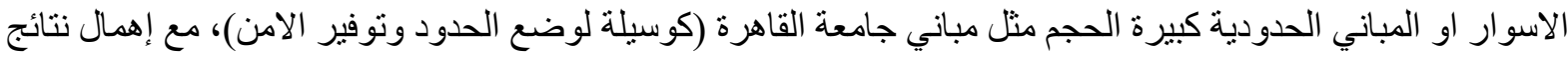

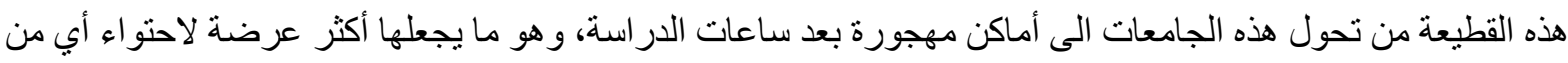

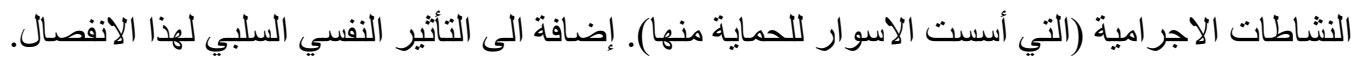

ـ ـ ـ ــ. توزيع الخدمات بحرم الجامعة: مع التوسع في انشاء الفروع وتحويلها بعد ذلك الى جامعات مستقلة، و الاعتماد

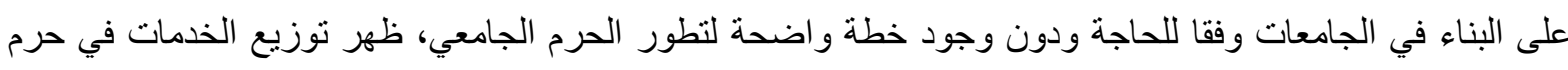

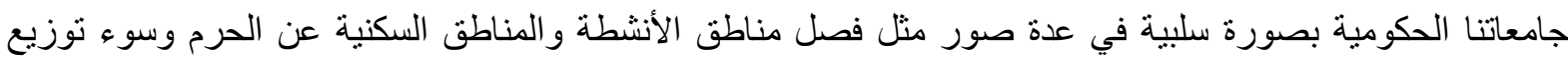
المناطق الخدمية.

ـ ـ ـ ـ. الصورة البصرية لحرم الجامعة: تمثل الصورة البصرية محفزا أساسيا في استخدام الفراغات والبيئات

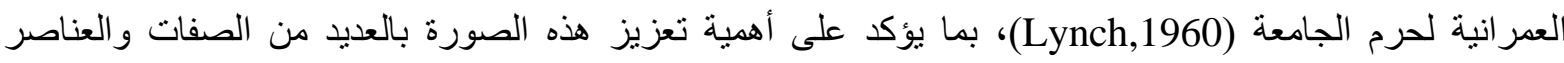

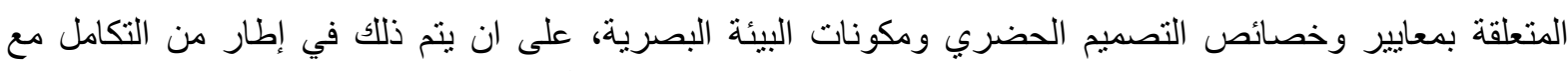
عناصر تنسيق الموقع المختلفة بما يساعد على خلق صورة بصرية أكثر شمولاً وحيوية (Cullen, 1971). ع-r. التحديات الإدارية التشغيلية للجامعة: والخاصة بتطور اعداد المقبولين بالحرم الجامعي مقارنة بالمساحة

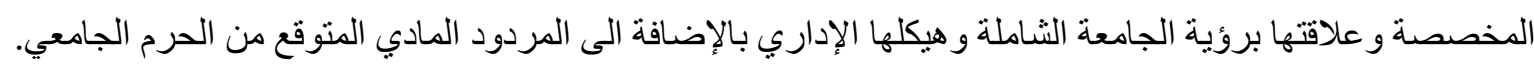
ع ـ - ا. ـ الروية الثاملة للجامعة: تمثل رؤية الجامعة في تطوير خطط التعليم و الحرم الجامعي بكافة خدماته ومبانيه

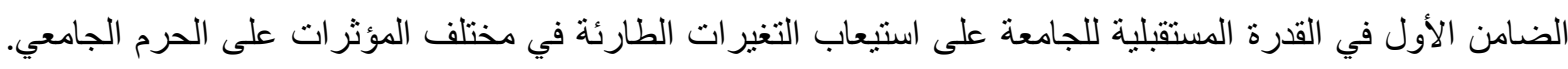

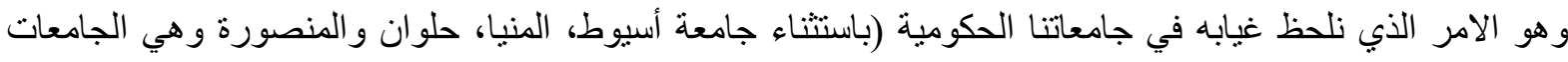

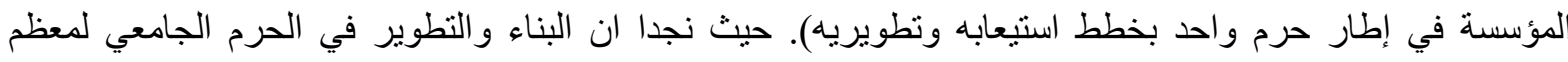

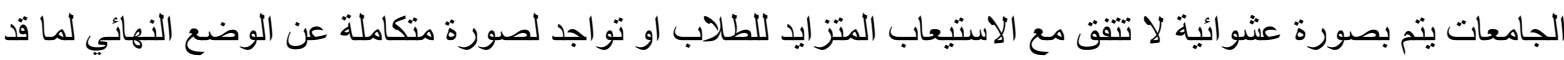

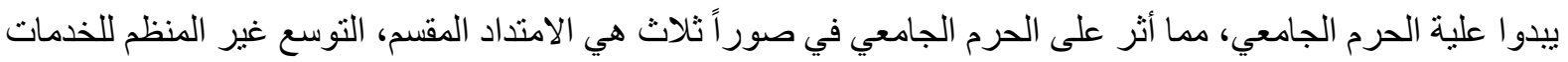

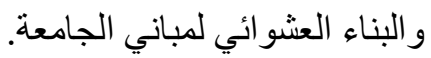

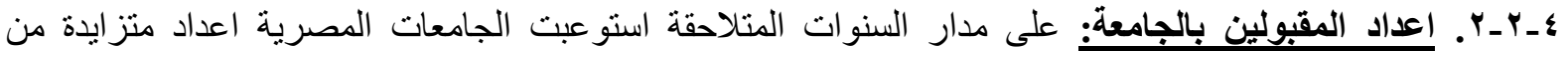

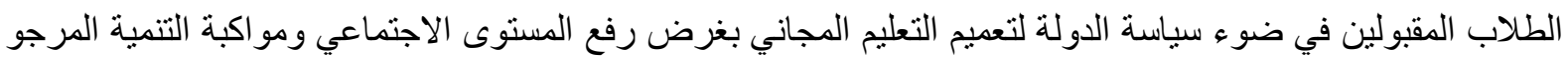

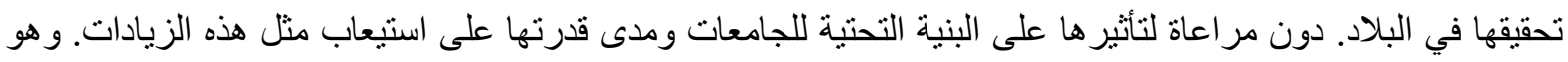

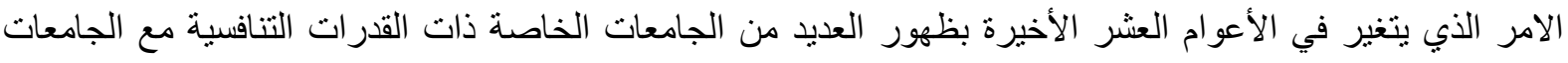

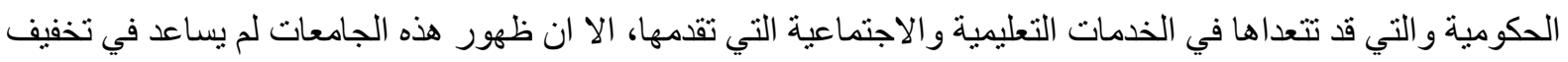

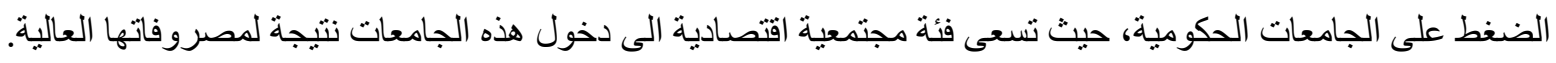
هذه الزيادة بالإضافة الى تأثير ها على البنية التحتية للجامعة فإنها تؤثر على المساحة المخصصة لكل طالب في الحرم

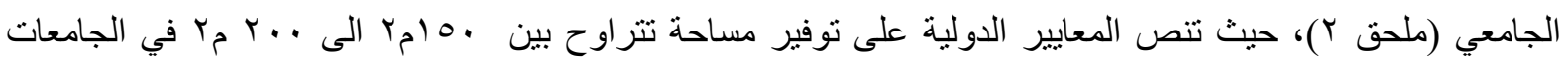

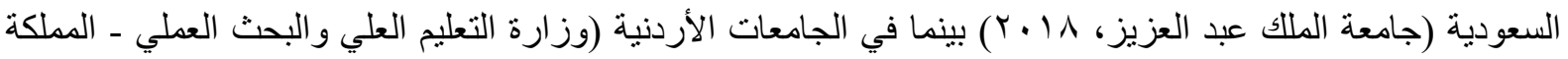

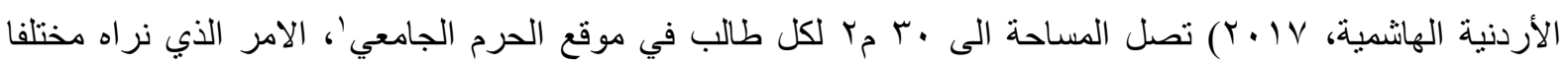

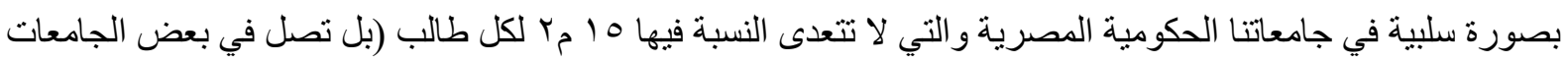

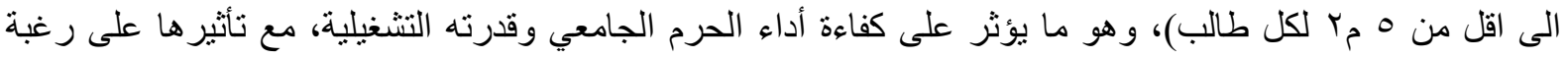

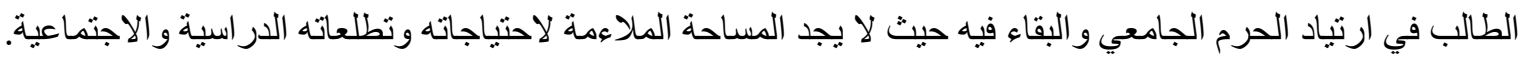




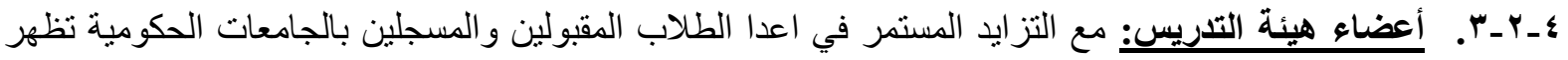

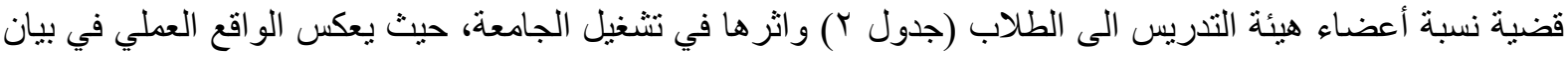

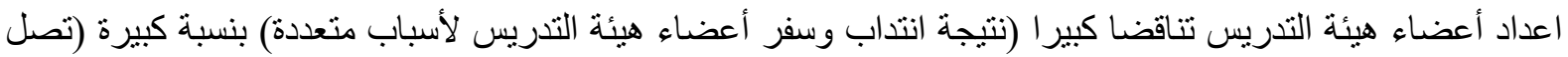

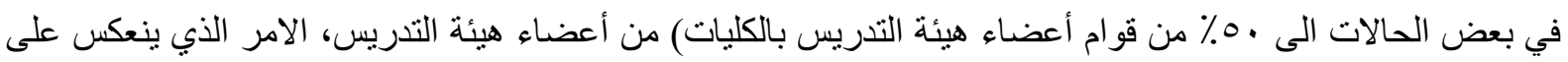

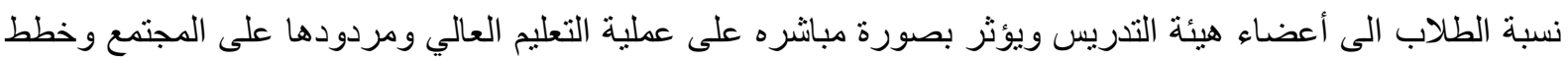

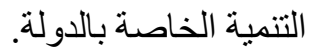

جدول r نسبة الطلاب الى أعضاء هيئة التدريس

\begin{tabular}{|c|c|c|c|}
\hline \multicolumn{2}{|c|}{ المعدلات المصرية (Abdel Kader, 2000) } & \multicolumn{2}{|r|}{ المعدلات العالمية (Sameh, 2000) } \\
\hline & & $1: 20$ & 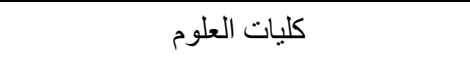 \\
\hline & & $1: 15$ & كليات الهندسة، الطب، الصيدلة و التمريض \\
\hline $1: 50$ & الكليات العملية العية & $1: 18$ & متوسط الكليات العملية \\
\hline $1: 100$ & 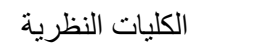 & $1: 30$ & كليات الفنون، العلوم الاجتماعية و الإنسانية \\
\hline
\end{tabular}

• تظهر الأرقام التفاوت الكبير بين ما تتص علية المعايير المصرية من نسب ـ تصل الى ضعف ما تتص علية المعايير الدولية
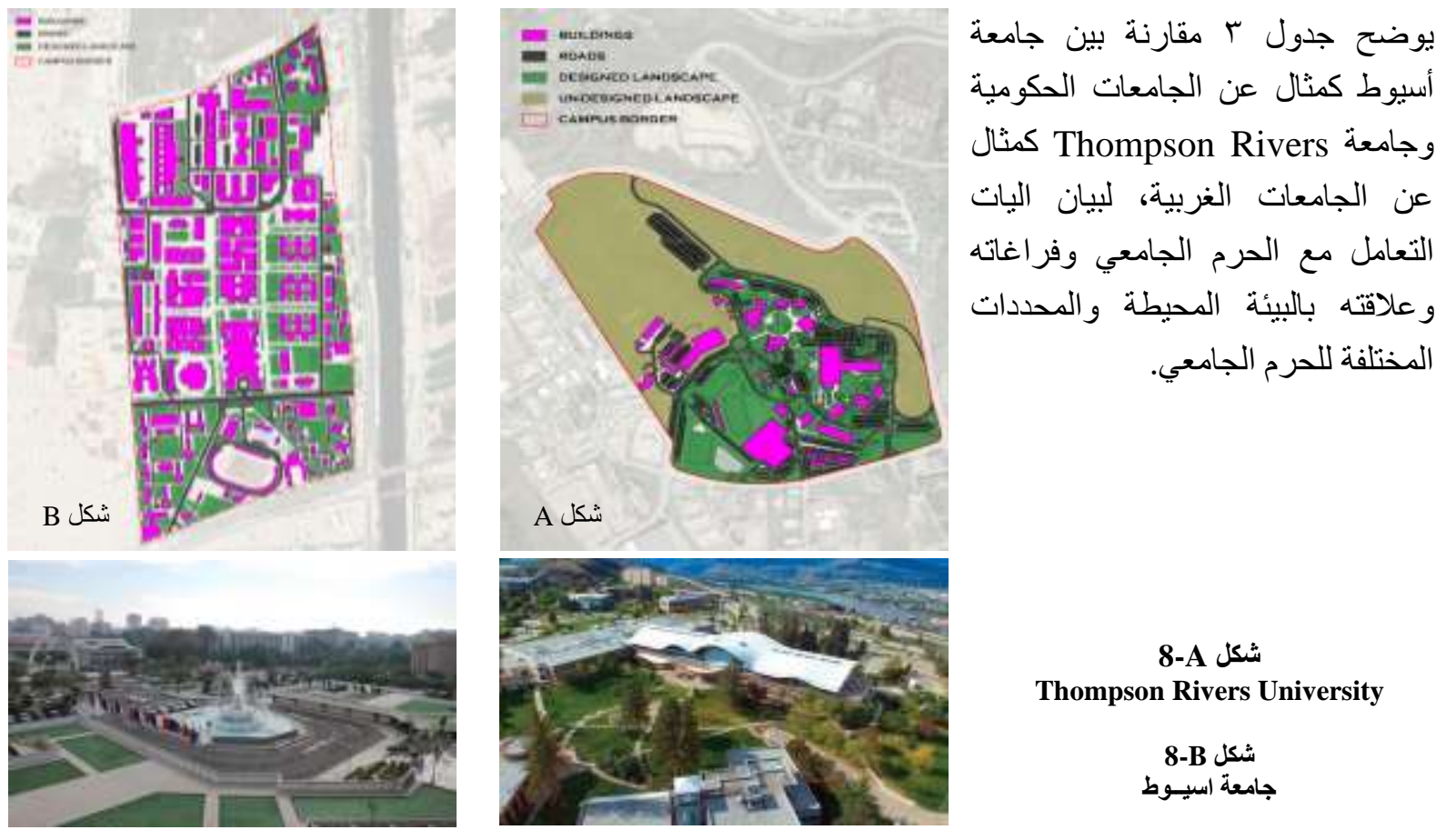

يوضح جدول r مقارنة بين جامعة أسيوط كمثال عن الجامعات الحكومية بردية وجامعة Thompson Rivers كمثنال عن الجامعات الغربية، لبيان اليات التعامل مع الحرم الجامعي وفر اغاته و علاقته بالبيئة المحيطة و المحددات

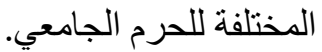

جدول r اليات التعامل مع الحرم الجامعي بالجامعات الغربية (Thompson Rivers University) و الجامعات الحكومية المصرية (جامعة (أسيوط)

\begin{tabular}{|c|c|c|}
\hline جامعة اسيوط & $\begin{array}{c}\text { Thompson Rivers } \\
\text { University }\end{array}$ & \\
\hline \multicolumn{3}{|c|}{ معلومـــات وحقائستق عامة } \\
\hline 962,459 & 856,780 & المساحة الاجمالية للحرم الجامعي/م؟ \\
\hline 285,205 & 70,250 & المساحة البنائية/مج \\
\hline $29.6 \%$ & $8.2 \%$ & النسبة البنائية \\
\hline 62,573 & 14,000 & عدد الطلاب المسجلين \\
\hline
\end{tabular}


الجامعات الحكومية المصرية "در اسة استقر ائية في نشأة وو اقع الجامعات الحكومية المصرية"

تابع جدول ؟ اليات التعامل مع الحرم الجامعي بالجامعات الغربية (Thompson Rivers University) والجامعات الحكومية المصرية (جامعة أسيوط)

\begin{tabular}{|c|c|c|}
\hline جامعة اسيوط & $\begin{array}{c}\text { Thompson Rivers } \\
\text { University }\end{array}$ & \\
\hline 10.8 & 56.2 & المساحة المتاحة لكل طالب في الفراغات الخارجية/مج \\
\hline 164,475 & 145,000 & مساحة الطرق ومواقف انتظار السيارات/مج \\
\hline$\%$ iv & $\% 1 v$ & نسبة الطرق ومواقف انتظار السيارات بالموقع العام \\
\hline 183,400 & 590,000 & مساحة المسطحات الخضراء/مج \\
\hline$\% 19$ & $\% 99$ & نسبة المناطق الخضر اء بالموقع العام \\
\hline \multicolumn{3}{|c|}{ اليات التعامل مع تعديات الحرم الجامعي } \\
\hline جمود الفكر التصميمي & تطوير الحرم بالاشتراك مع المستخدمين & الفكر التصميمي لحرم الجامعة \\
\hline الهمال في الصيانة وتحويل نشاط & الحفاظو التكامل بين عناصر تنسيق الموقع & حالة عناصر تتسيق الموقع \\
\hline ل ل ايوجد & 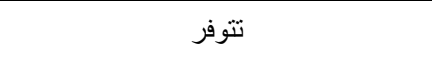 & إمكانية الامتداد المستقبلي \\
\hline الانفصال بالأسوار عن البيئة المحيطة & الاتصال بالبيئة المحيطة & تكامل حرم الجامعة والبيئة المحيطة \\
\hline 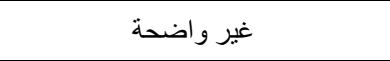 & تو افر منطقة خدمات مركزية & توزيع الخدمات بحرم الجامعة \\
\hline غير و اضحة & سيطرة عناصر تنسيق الموقع & الصورة البصرية لحرم الجامعة \\
\hline
\end{tabular}

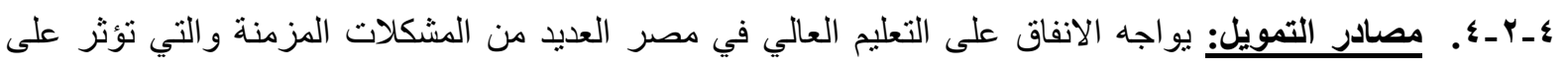

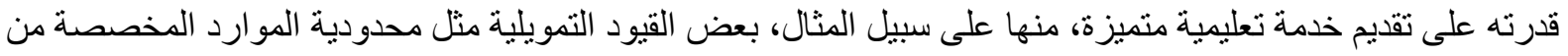

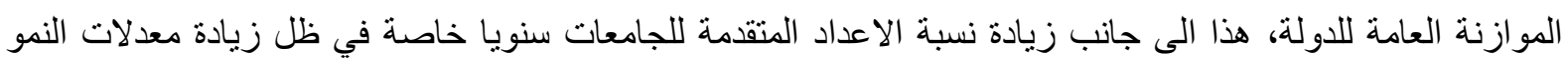

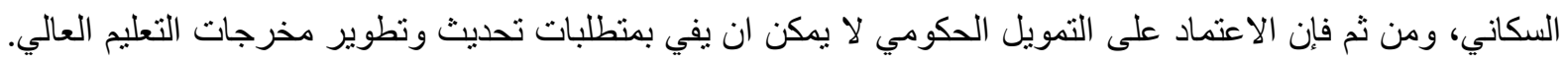

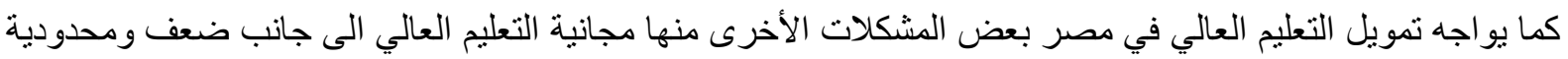
التمويل الخارجي.

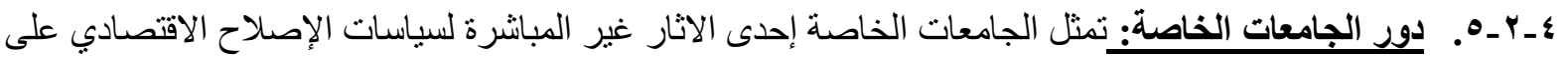

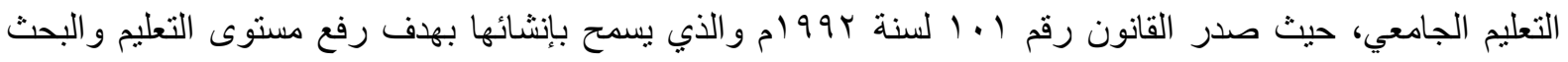

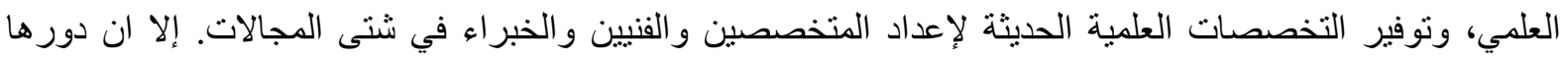

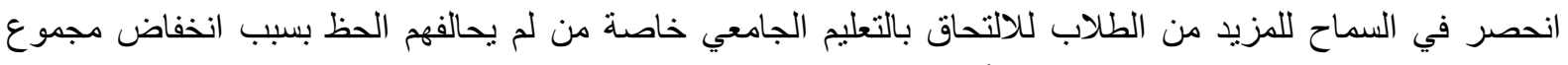

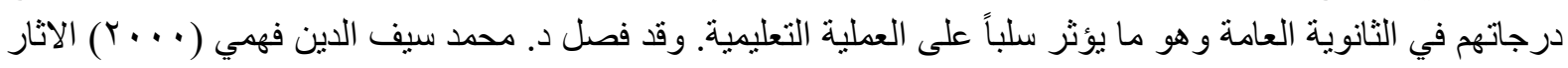
السبيه لها على مجتمع التعليم العالي كما يلي: لمانية

استهداف بعضاً منها الربح مما يدفعها لاستخدام أسليب جذب للطلاب قد لا تتوفر فيها المقاييس و المو اصفات

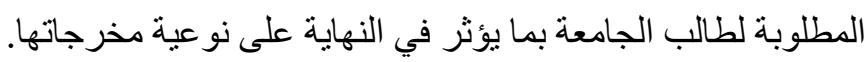

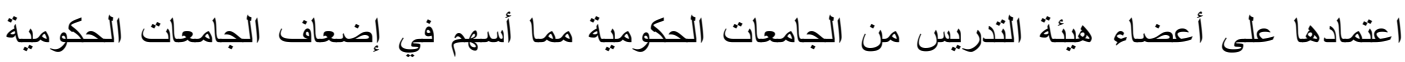

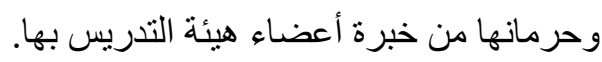
انها قد لا تقدم بر امج تعليمية تلبي الاحتياجات الحقيقية للمجتمع حيث قد تهمل بعض اعضئ التخصصات ذات الكات الكلفة العالية وتهنم بتقديم بر امج قليلة الكلفة.

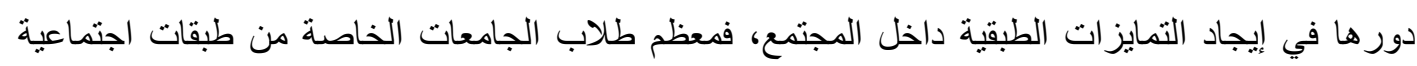

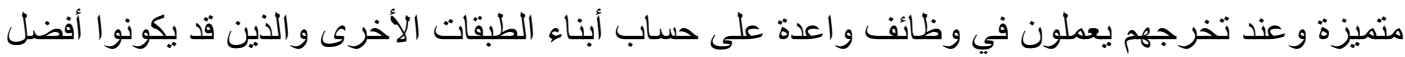
منهم في الإمكانات و القدرات. 


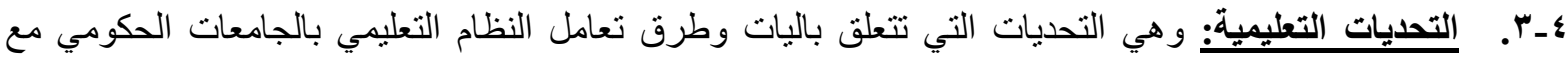

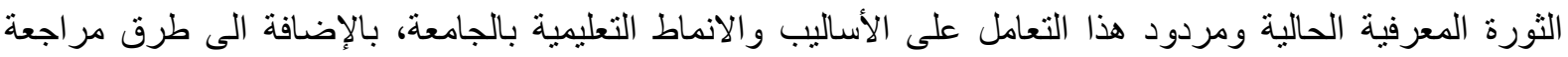

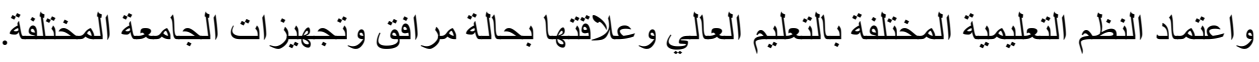

ـ ـــــــإدارة المعرفة: يمثل تصنيف المعرفة (الصريحة و الضمنية) تحديا رئيسيا في اليات التعامل مع الحرم الجامعي

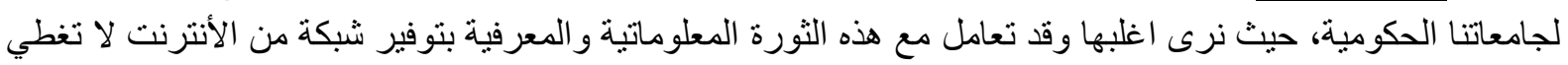

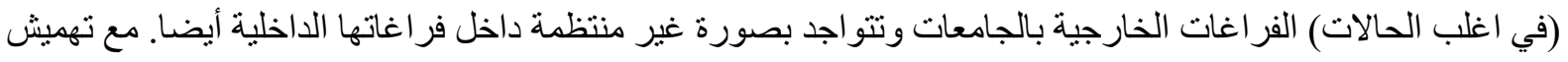

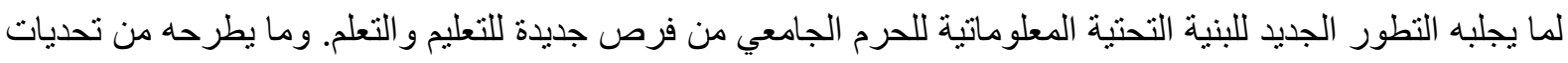

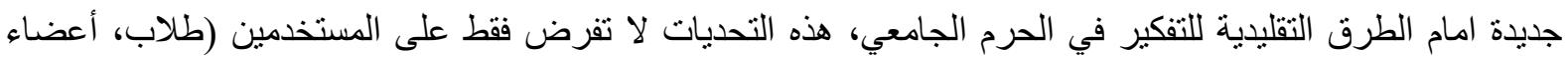
هيئة تدريس و اداريين) بل تمند الى النطاق المجنمعي الاوسع.

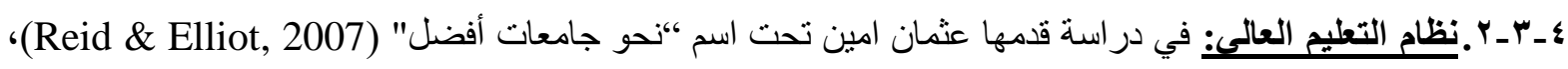

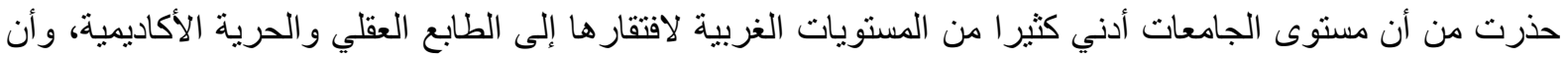

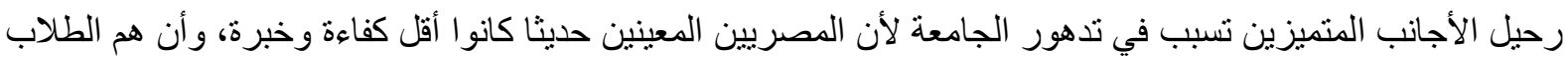

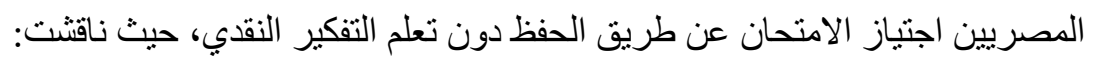

تمتع الجامعات المصرية بقدر ضئيل جدا من الاستقلالية.

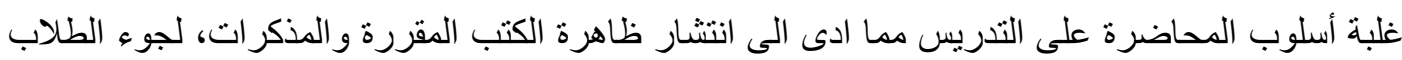

إلى حفظ المعلومات استعدادا للامتحانات، هبوط النابن الاستفادة من التطبيقات العملية.

جداول توزيع الدروس المكتظة بالمواد والتي لا تسمح للطلاب بمزاولة الأنشطة الرياضية والثقافية

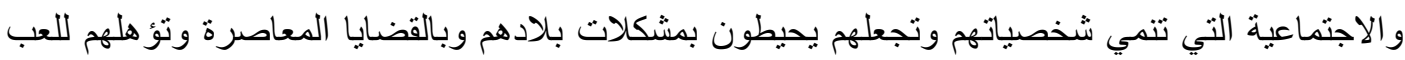

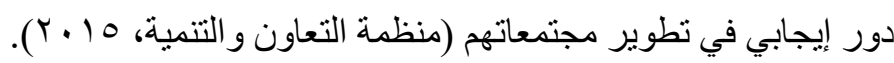

$$
\text { غياب الاستر اتيجية الو اضحة للبحث العلمي. }
$$

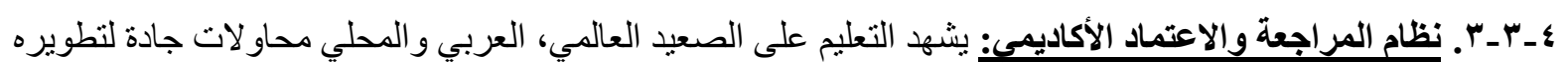

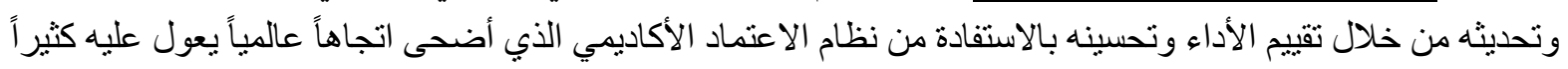

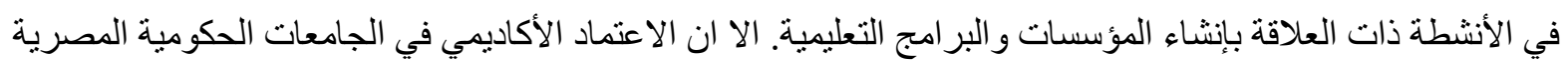

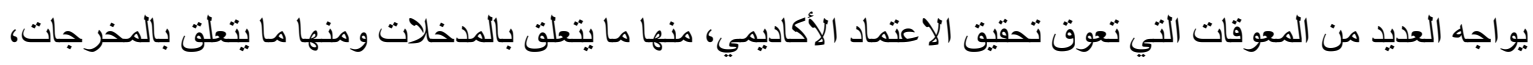
كما يلي:

ضعف التحديد الواضح و الدقيق لمعايير الاعتماد الأكاديمي ومؤشر اتها من قبل هيئات الاعتماد.

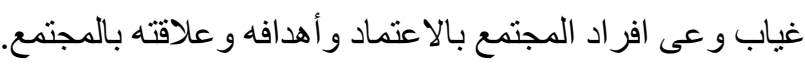

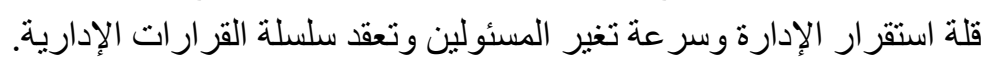

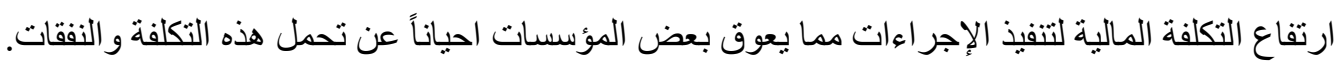

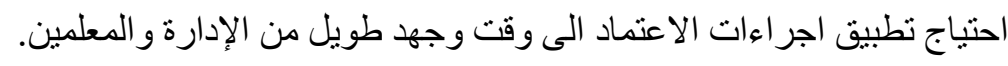

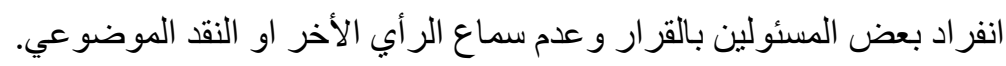

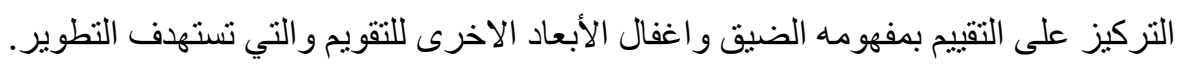

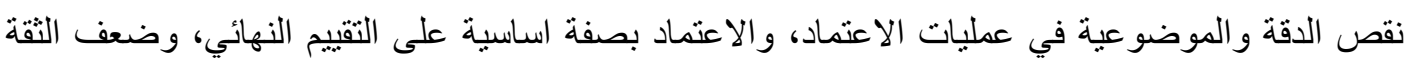
المتبادلة بين الأفر اد. 
ضعف نظام المعلوماتية في المجال التربوي وعدم التنسيق بين مصادر المعلومات و انعكاساتها السلبية في عملية الاعتماد. الآراء المتغيرة للخبراء المشاركين في عمليات الاعتماد الأكاديمي تجعل هذه العمليات احياناً غير

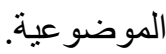
بعض هيئات الاعتماد الأكاديمي قد تتعرض داخلياً او خارجيا لبعض الضغوط. عدم وجود فلسفة عامة واستر اتيجية مستقبلية للمؤسسة التعليمبة. عدم وجود نظم منطورة لإعداد اعضاء هيئة التدريس بما يكفل تنمية قدر اتهم.

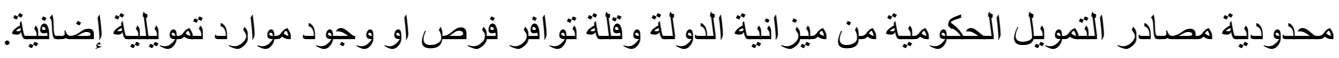
مقاومة التغيير وضعف الاتصال. قصور فهم نظام الاعتماد و عدم رغبة الإداريين في تطبيقها.

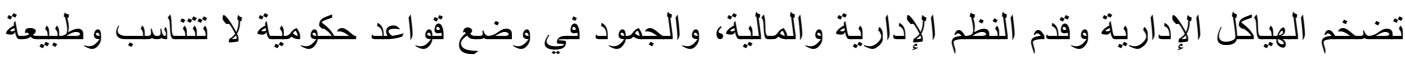
المؤسسات التعليمية العلمية و البحثية.

؛ ـ ـ ـ ـ الجامعات الحكومية المصرية و التصنيف العالمى: يعد خروج الكثير من الجامعات الحكومية من التصنيف

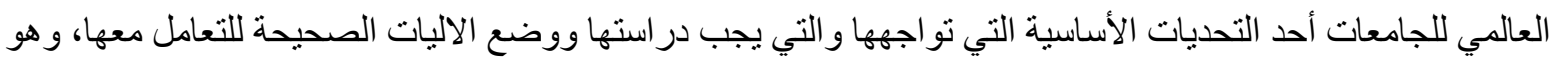

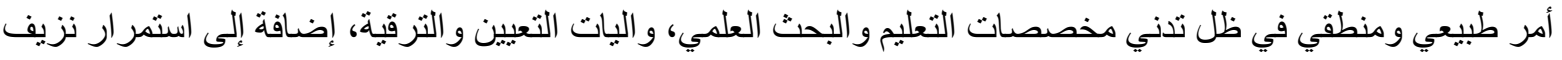

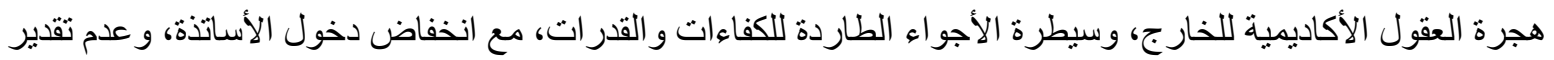

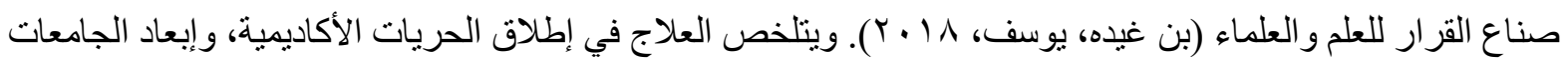

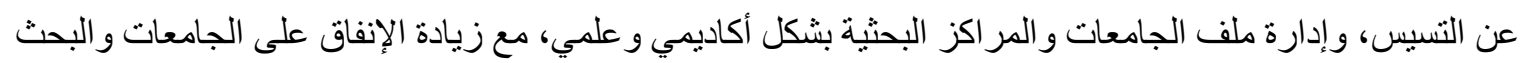

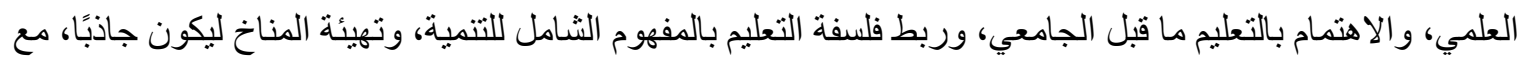

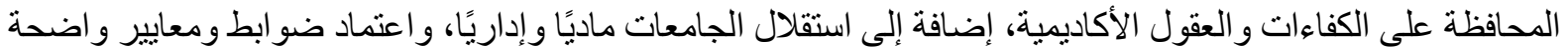
لترقية الأساتذة، تعتمد على الموضو عية، و الثفافية، و القدرة، والكية الكفاءة.

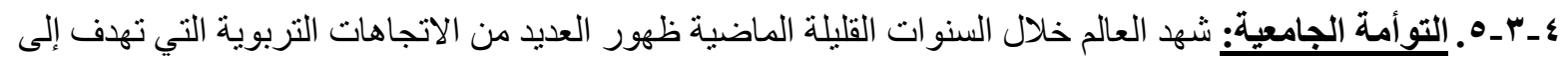

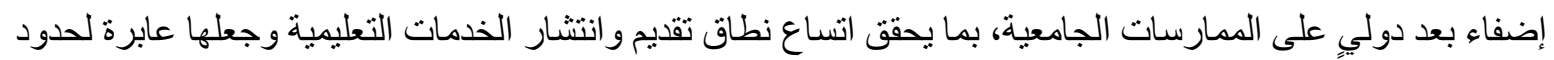

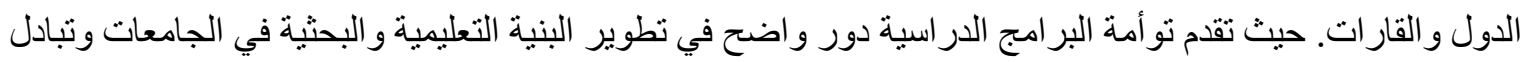

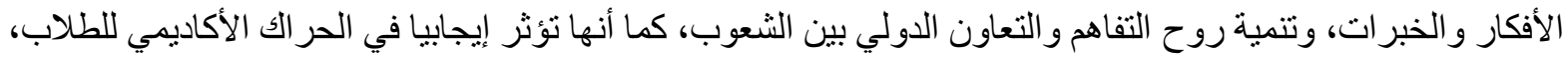

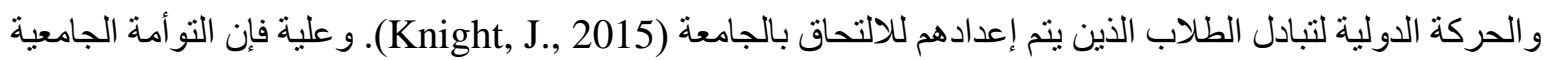

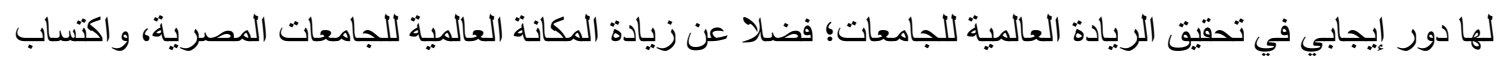

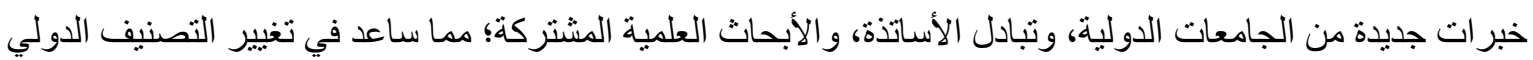

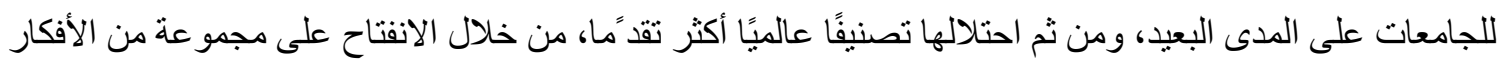
الجديدة عبر أوسع التخصصات (Paul, S., 2014).

\section{•.}

يمكن تلخيص نتائج الدر اسة فيما يلي:

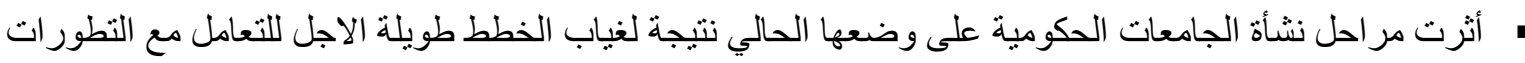

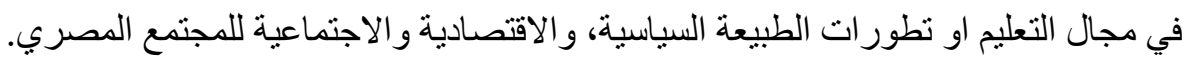

" سعي الجامعة إلى تأسيس بيئة تعليمية ناجحة يعد الركيزة الأساسية للرؤية الثناملة التي قد تنتهجها، حيث يتأثر بكافة التحديات التي تو اجههاويؤثر على الطبيعية التصميمية التي تهدف اليها الجامعة. 
الجامعات الحكومية المصرية "در اسة استقر ائية في نشأة وو اقع الجامعات الحكومية المصرية"

تمثل التحديات الإدارية / التشغيلية والتحديات التعليمية حالة خاصة بالجامعات الحكومية المصرية لغياب خطط التعامل مع مو اطن القوة في منظومة التعليم و الجامعات الحكومية المصرية. يجب ان تشمل خطط و أهداف تطوير الجامعات الحكومية المصرية اليات للتعامل مع كافة تحدياتها، حيث تمثل هذه

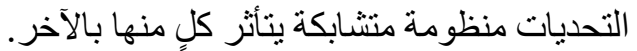

T.

تكمن أهمية نتائج الدر اسة في طرحها لرؤية شاملة عن مواطن القوة والتحديات التي تو اجه الجامعات الحكومية المصرية،

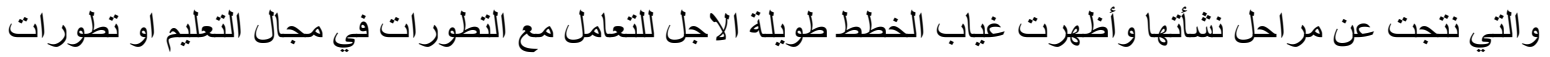

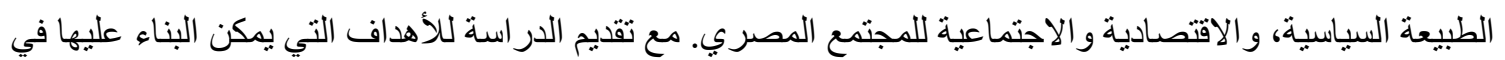

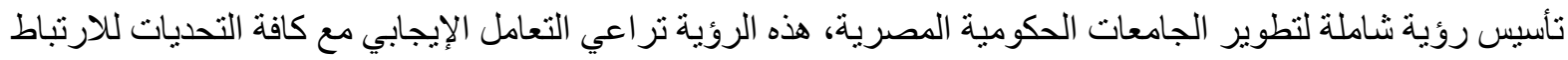

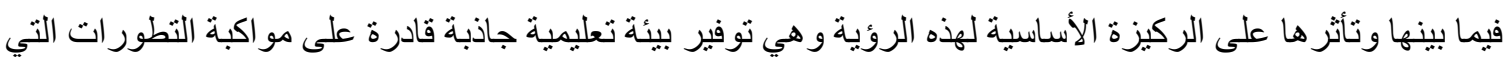
تحدث على المستويات التعليمية، و السياسية، و الاقتصادية، و الاجتماعية.

V

• العمل عل تأسيس رؤية شاملة لتطوير الجامعات الحكومية المصرية تسعى إلى توفير بيئة تعليمية جاذبة قادرة على مو اكبة التطور ات التي تحدث على المستويات التعليمية، والسياسية، و الاقتصادية، والاجتماعية. • مر اجعة اليات اختيار مواقع تأسيس الجامعات الحكومية وارتباطها بأعداد المقبولين ومناطق التغطية لكل حرم بما يحد من عدة مشاكل منها الاغتراب.

تنمية دور الجامعات الحكومية في تعزيز الثعور المجتمعي بما يضمن توفير الموارد المالية اللازمة بالإضافة إلى تفعيل دور ها في تلبية احتياجات المجتمع. • تطوير اليات تصميم الحرم الجامعي للجامعات الحكومية المصرية لتو اكب التطور ات المختلفة للجامعات على مستوى العالم وبما يتلاءم مع و اقعنا المصرئ لمعري.

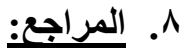

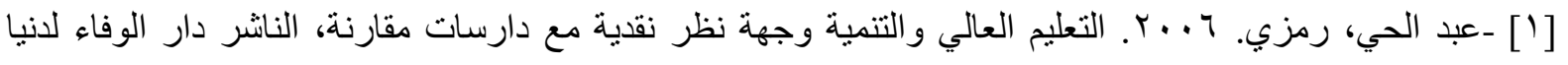

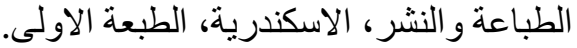

[2] -Turner 'Paul V. (1996). Joseph Ramée: International Architect of the Revolutionary Era. Cambridge: Cambridge University Press. P.190.

[3] -Mitchell, W. J., \& Vest, C. M. (2007). Imagining MIT: Designing a campus for the twenty-first century. Cambridge, MA: MIT Press.

[4] -Malden, H. (1835). On the Origin of Universities and Academical Degrees. Harvard: J. Taylor, P14.

$$
\text { [0] المؤتمر القومي للتعليم العالي، الخطة الاستر اتيجية لتطوير منظومة التعليم العالي، مصر، ... ؟. }
$$

[6] -Anderson, R. (2010). The 'Idea of a University' today. History \& Policy.

[7] -Pearce, Martin. (2001). University builders. London: John Wiley and sons, p.10.

[8] -Lynch, K. (1960). The image of the city. Cambridge, Mass: MIT Press. 
[9] -Cullen, G. (1971). The concise townscape. New York: Van Nostrand Reinhold Company.

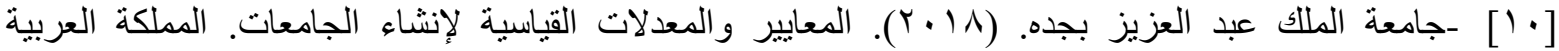

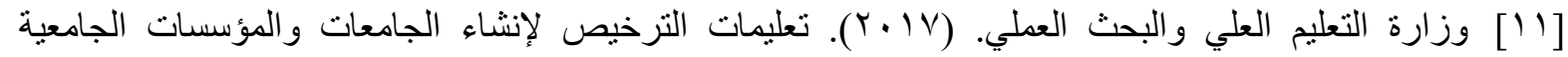
الخاصة و إجر اءاته. المملكة الأردنية الهانشمية.

[12] -Sameh, Salah. (2000). Quality Assurance in University Education, Journal of Engineering Education Vo1.10 No 2, Great Britain.

[13] -Abdel Kader, Mourad. ( $\uparrow . . \cdot$ ). Standards of private university faculties in Egypt. Ain Shams University, Egypt.

[14] -Reid, Donald \& Elliot, Colla. (2007). Conflicted Antiquities: Egyptology, Egyptomania, Egyptian Modernity. Durham and London: Duke University Press.

[10] ـ ـمنظمة التعاون و التنمية في الميدان الاقتصادي والبنك الدولي. (10 • ب)، مراجعات لسياسات التعليم الوطنية:

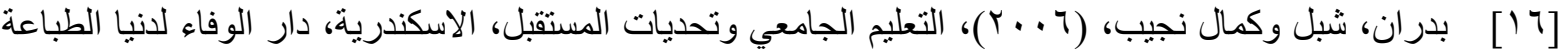
و النشر.

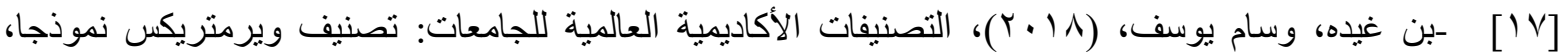

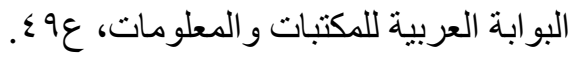

[18] -Knight, J. (2015): Monitoring the quality and Process of Internationalization Journal of Studies in International Education, 5(3).

[19] -Paul, S. (2014): Internationalization of Higher Education: Strategic Implications. Economic \& political weekly, Vol X 9. 
الجامعات الحكومية المصرية "در اسة استقر ائية في نشأة وو اقع الجامعات الحكومية المصرية"

ملحق 1 ـ تحديات الجامعات الحكومية المصرية

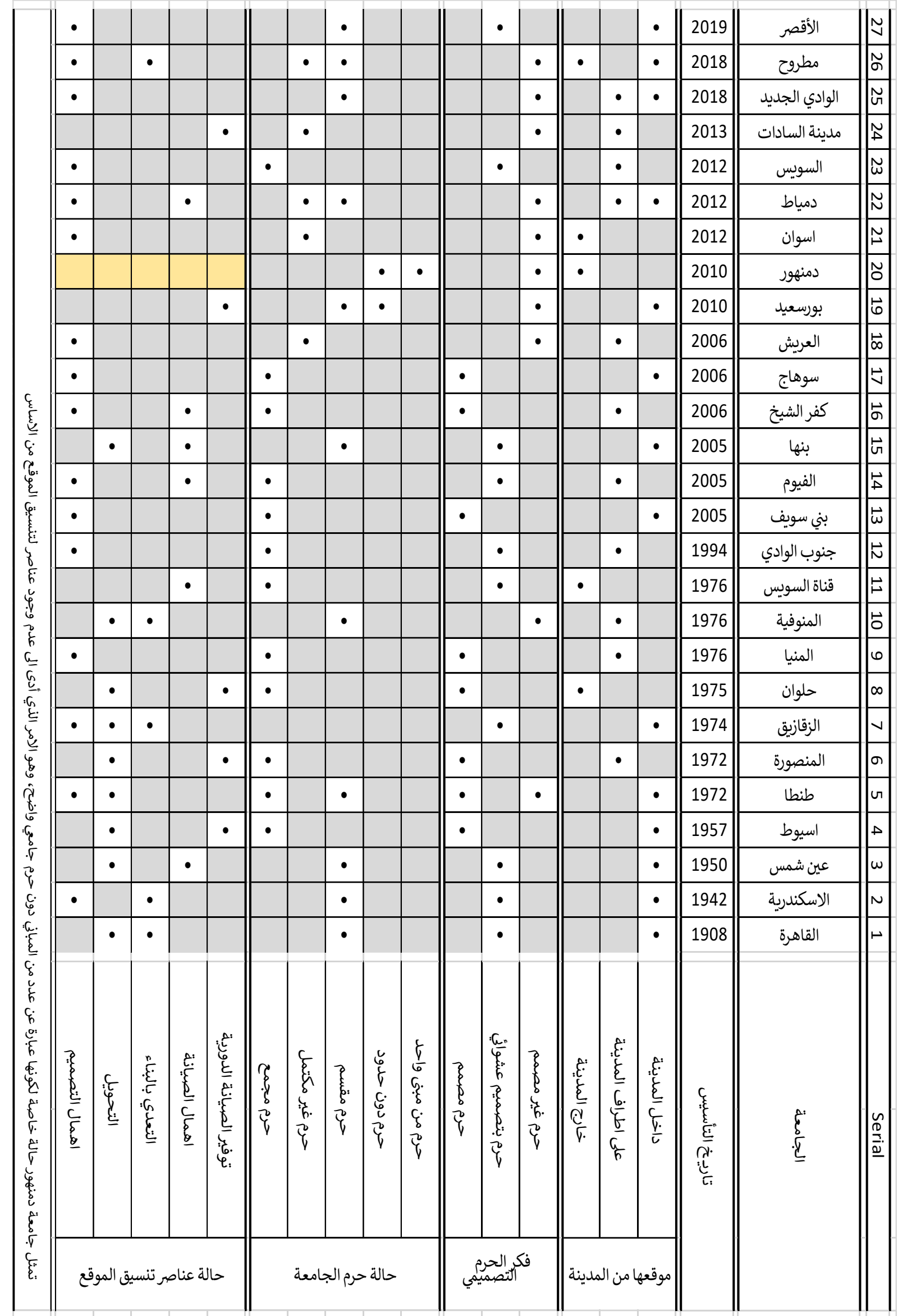


الجامعات الحكومية المصرية "در اسة استقر ائية في نشأة وو اقع الجامعات الحكومية المصرية"

تابع ملحق 1 ـ تحديات الجامعات الحكومية المصرية
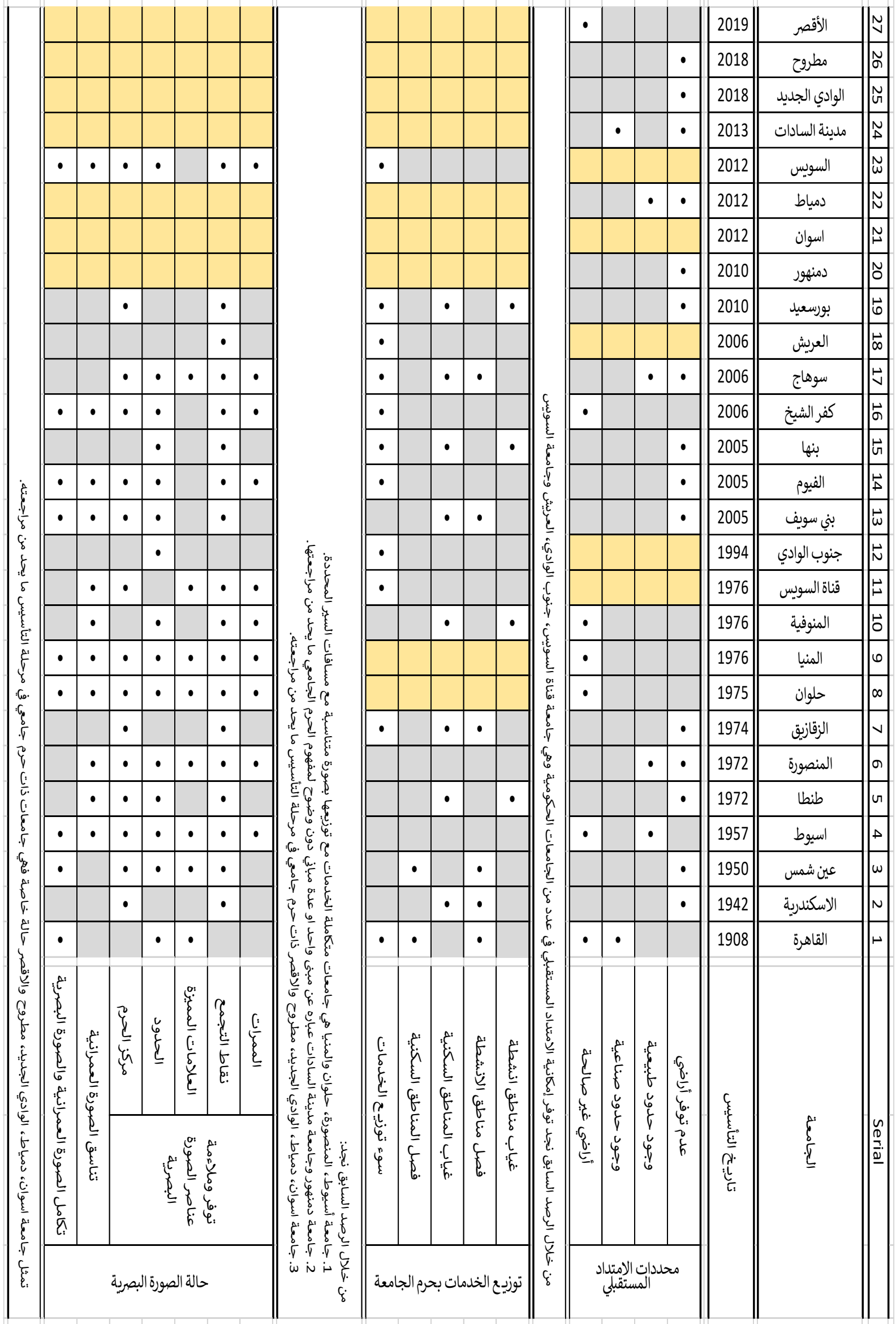
الجامعات الحكومية المصرية "در اسة استقر ائية في نشأة وو اقع الجامعات الحكومية المصرية"

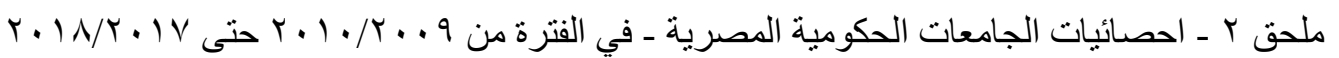

\begin{tabular}{|c|c|c|c|c|c|c|c|c|c|c|c|c|c|c|c|c|c|c|c|c|c|}
\hline$\sim \sqrt[v]{\partial}$ & के & & & $N$ & ஜे & & $\vec{\infty}$ & $\mid \overrightarrow{\vec{\omega}}$ & & & $\vec{V}$ & s. & & $|\widetilde{v}| \dot{a}$ & \begin{tabular}{c|c}
$\sim$ \\
$o$ \\
$c$
\end{tabular} & & $\underline{\sim}$ & ب̂. & & & \\
\hline $\begin{array}{lll}\omega & \infty \\
\oplus & \stackrel{\infty}{\omega}\end{array}$ & 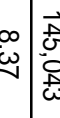 & $\frac{\vec{N}}{\vec{N}}$ & & $v$ & 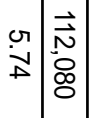 & 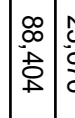 & 筩|| & $\begin{array}{l}0 \\
\grave{d}\end{array}$ & 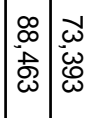 & \begin{tabular}{|l|}
$\vec{v}$ \\
$\stackrel{\vec{d}}{0}$
\end{tabular} & \begin{tabular}{l|l}
$\vec{\omega}$ & $\omega$ \\
$\ddot{\theta}$
\end{tabular} & 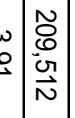 & 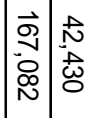 & $\omega \omega$ & \begin{tabular}{c|c|c}
$\sim$ & $\vec{\infty}$ \\
$\stackrel{\infty}{\infty}$ & $\stackrel{\sim}{\infty}$ \\
$\vec{\sigma}$ &
\end{tabular} & 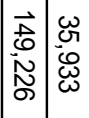 & $\vec{\sigma}$ & 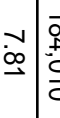 & 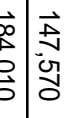 & $\mid$ & $\underset{\infty}{\vec{\Delta}}$ \\
\hline 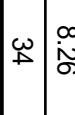 & 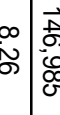 & 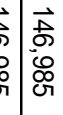 & & 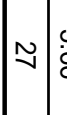 & \begin{tabular}{c|c|c|}
$\stackrel{\vec{\omega}}{\omega}$ \\
$\stackrel{\vec{\omega}}{\vec{\sigma}}$ \\
$\vec{\sigma}$
\end{tabular} & | & $\widetilde{\omega}$ & $\stackrel{0}{=}$ & 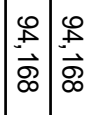 & & 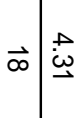 & 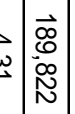 & $\mid$ & $\omega \mid \hat{\theta}$ & 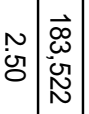 & 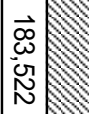 & $\underline{\sim}$ & 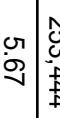 & 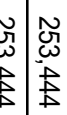 & & $\overrightarrow{\vec{\partial}}$ \\
\hline$\stackrel{\omega}{\omega} \underset{\partial}{\infty}$ & 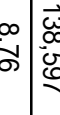 & \begin{tabular}{l|l}
$\vdots$ & $\vec{\omega}$ \\
$\vdots$ & 0 \\
0 & 0 \\
0 & 0 \\
$\vdots$ &
\end{tabular} & & $\tilde{N}$ & 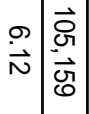 & 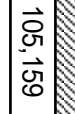 & $\approx$ & $\mid$\begin{tabular}{|l|}
$\vec{O}$ \\
$\dot{\omega}$ \\
$\vec{v}$
\end{tabular} & 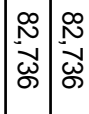 & & $\vec{\infty} \underset{\dot{\infty}}{\stackrel{\vec{\sim}}{\vec{\sim}}}$ & 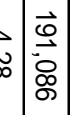 & $\mid \begin{array}{c}\overrightarrow{0} \\
\vec{\circ} \\
\stackrel{\circ}{o}\end{array}$ & $\omega$ & 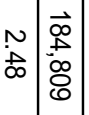 & 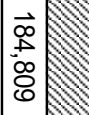 & $N$ & 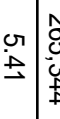 & 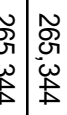 & & $\begin{array}{l}\vec{v} \\
\vec{\sigma}\end{array}$ \\
\hline$\cong \cong$ & 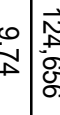 & 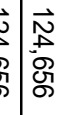 & & $N$ & 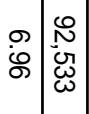 & 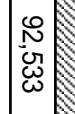 & $\vec{\omega}$ & $\mid \overrightarrow{\overrightarrow{\vec{\Delta}}}$ & 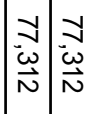 & & $\vec{V} \underset{\vec{f}}{\vec{c}}$ & 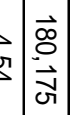 & $\mid \begin{array}{l}\overrightarrow{0} \\
\stackrel{\vec{v}}{\vec{v}}\end{array}$ & $\approx)$ & 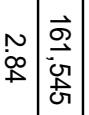 & $\left|\begin{array}{|l}\vec{a} \\
\vec{c} \\
\vec{c}\end{array}\right|$ & $\overrightarrow{0}$ & 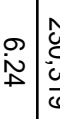 & 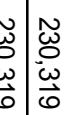 & & $\frac{\vec{A}}{\vec{v}}$ \\
\hline$\simeq \begin{array}{ll}\vec{\omega} \\
\vec{\sigma}\end{array}$ & 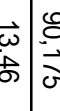 & $\begin{array}{ll}0 \\
\overrightarrow{3}\end{array}$ & & $\vec{\omega}$ & \begin{tabular}{l|l}
$\infty$ & 0 \\
$\vec{\sigma}$ & $\infty$ \\
$\dot{\sigma}$ & 0 \\
0 & 0
\end{tabular} & $\mid \begin{array}{l}\infty \\
\infty \\
o \\
0 \\
y\end{array}$ & $\vec{\sigma}$ & $\left|\begin{array}{c|}\vec{\omega} \\
\dot{\sigma} \\
\end{array}\right|$ & 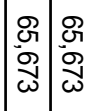 & & $\vec{\omega} \mid \stackrel{\vec{N}}{ }$ & 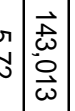 & 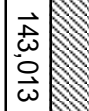 & $\approx$ & 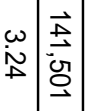 & $\left|\begin{array}{|l}\overrightarrow{+} \\
\overrightarrow{0} \\
0\end{array}\right|$ & $\approx$ & 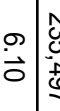 & 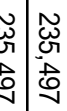 & & $\frac{\vec{\omega}}{\vec{\Phi}}$ \\
\hline$N \vec{N}$ & 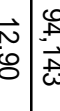 & 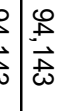 & & $\vec{\omega}$ & 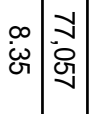 & $\begin{array}{l}3 \\
0 \\
0 \\
\text { a }\end{array}$ & $\vec{\sigma}$ & $\left|\begin{array}{l}\vec{\omega} \\
\overrightarrow{\vec{v}}\end{array}\right|$ & 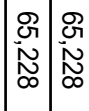 & & 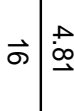 & 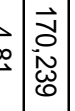 & $\vec{D}$ & $\tilde{\omega} \frac{0}{\alpha}$ & 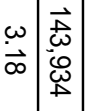 & $\mid$\begin{tabular}{|l}
$\vec{\omega}$ \\
$\omega$ \\
$\omega$ \\
$\dagger$
\end{tabular} & $\vec{\bullet}$ & 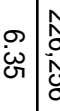 & 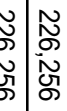 & & $\frac{\vec{v}}{\omega}$ \\
\hline$N \vec{\omega}$ & 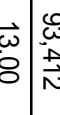 & 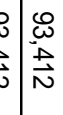 & & $\overrightarrow{0}$ & 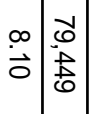 & $\begin{array}{l}0 \\
0 \\
\text { 类 }\end{array}$ & के & $\left|\begin{array}{c|}\vec{\omega} \\
\dot{\omega} \\
\tilde{\omega}\end{array}\right|$ & 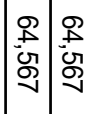 & & $\vec{ज} \mid \vec{c}$ & 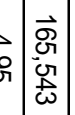 & $\left|\begin{array}{|l}\overrightarrow{9} \\
- \\
0 \\
\omega \\
\omega\end{array}\right|$ & $\approx \tilde{\omega}$ & 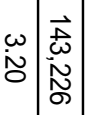 & $\left|\begin{array}{|c}\vec{c} \\
\omega \\
\tilde{N} \\
\widetilde{O}\end{array}\right|$ & $\vec{\emptyset}$ & 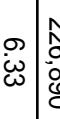 & 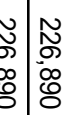 & & $\underset{\vec{N}}{\vec{\Delta}}$ \\
\hline$\tilde{\omega} \overrightarrow{\tilde{g}}$ & 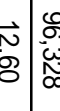 & 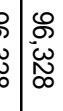 & & $\approx$ & 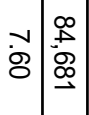 & $\mid \begin{array}{l}\infty \\
\stackrel{\infty}{a} \\
\stackrel{2}{\infty}\end{array}$ & $\overrightarrow{\text { जे }}$ & $\left|\begin{array}{l}\vec{\omega} \\
\ddot{\omega}\end{array}\right|$ & 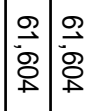 & & $\vec{\sigma} \underset{\dot{\infty}}{\stackrel{\dot{\phi}}{+}}$ & 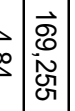 & $\left|\begin{array}{|l}\overrightarrow{8} \\
\tilde{W} \\
\mathrm{~m}\end{array}\right|$ & $\widetilde{\omega}$ & 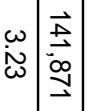 & $\left|\begin{array}{c|}\vec{f} \\
\vec{\infty} \\
\vec{y}\end{array}\right|$ & $\approx$ & ب) & 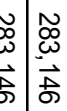 & & $\stackrel{\vec{O}}{\Xi}$ \\
\hline $\mathbb{N}$ & 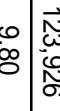 & 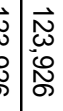 & & $\widetilde{\omega}$ & 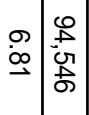 & $\begin{array}{l}0 \\
+ \\
0 \\
0 \\
0\end{array}$ & $\vec{v}$ & $\mid \begin{array}{l}\overrightarrow{\vec{a}} \\
\dot{\mathrm{o}} \\
\mathrm{v}\end{array}$ & 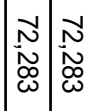 & & $\vec{\omega} \mid$\begin{tabular}{l|l}
$\omega$ \\
$i$ \\
$i$
\end{tabular} & 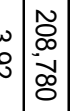 & $\left|\begin{array}{c}0 \\
0 \\
\infty \\
0 \\
0 \\
0\end{array}\right|$ & $\omega$ & 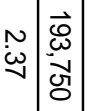 & $\mid$\begin{tabular}{|l}
$\overrightarrow{0}$ \\
$\omega$ \\
$\overrightarrow{0}$
\end{tabular} & $\tilde{v}$ & $\stackrel{\vec{y}}{\stackrel{y}{*}}$ & 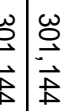 & & $\frac{8}{0}$ \\
\hline 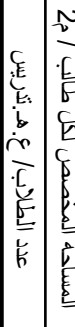 & 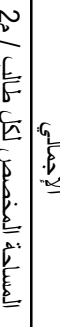 & & 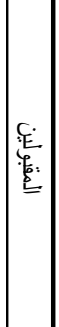 & 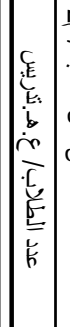 & 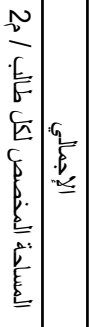 & 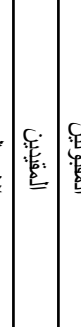 & 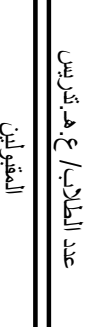 & 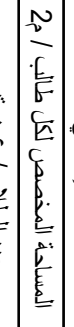 & & 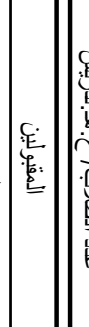 & 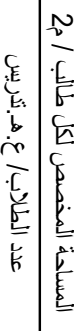 & 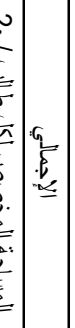 & 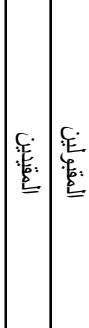 & 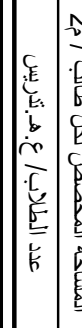 & 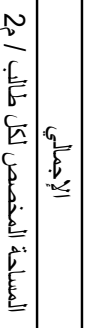 & 营: & 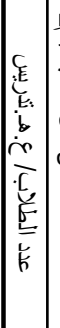 & 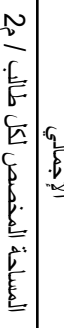 & & $\mid$ & \\
\hline 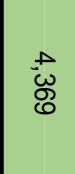 & 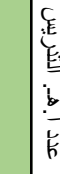 & 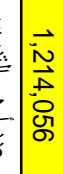 & E: & $\begin{array}{l}\vec{\sim} \\
\underset{\infty}{\infty}\end{array}$ & $\left|\begin{array}{l}\underline{E} \\
\underline{E} \\
b \\
b \\
\vec{b}\end{array}\right|$ & 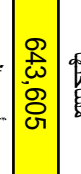 & & 芯 & 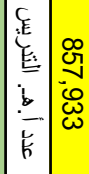 & E: & $\begin{array}{l}\overrightarrow{\overrightarrow{0}} \\
\bar{\phi}\end{array}$ & $\left|\begin{array}{l}\underline{E} \\
\underline{\underline{E}} \\
\dot{b} \\
\dot{b} \\
\bar{k}\end{array}\right|$ & 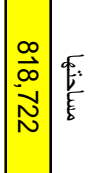 & 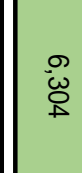 & $\left|\begin{array}{l}\bar{E} \\
\underline{E} \\
b \\
b \\
\bar{b}\end{array}\right|$ & 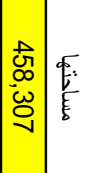 & $\stackrel{\vec{N}}{\vec{E}}$ & & 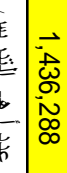 & E: & \\
\hline & 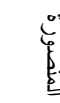 & & & & E: & & & & 占 & & & 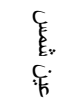 & & & 点 & & & & E: & & \\
\hline
\end{tabular}


الجامعات الحكومية المصرية "در اسة استقر ائية في نشأة وو اقع الجامعات الحكومية المصرية"

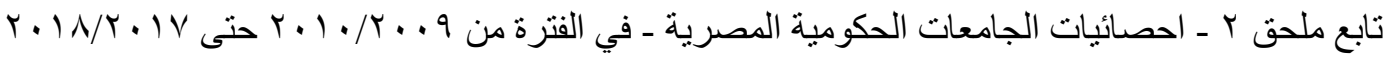

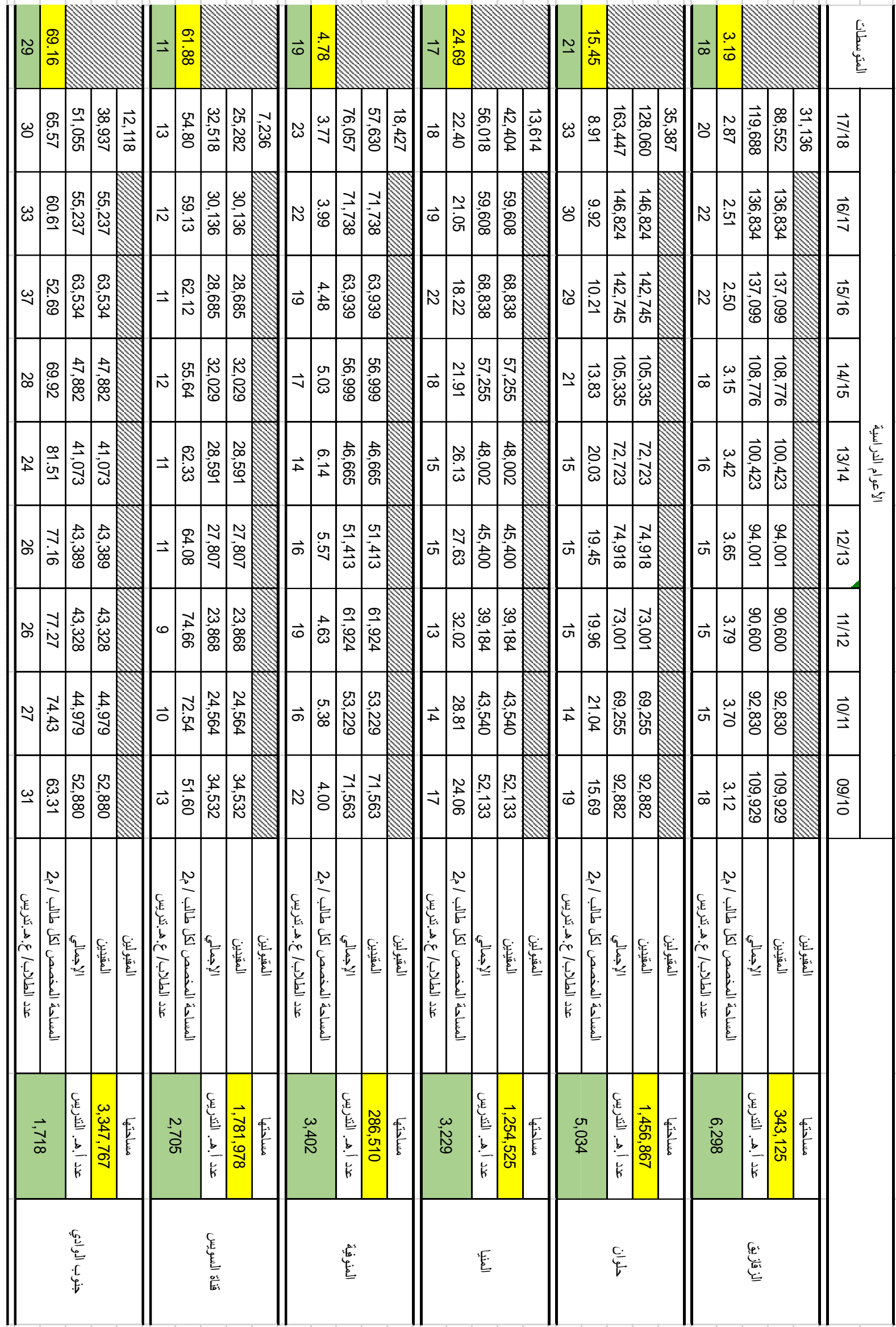


الجامعات الحكومية المصرية "در اسة استقر ائية في نشأة وو اقع الجامعات الحكومية المصرية"

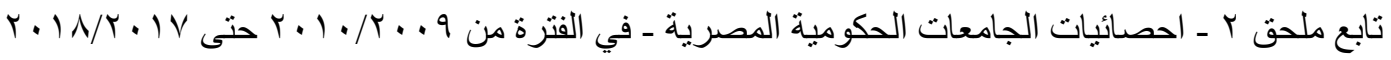

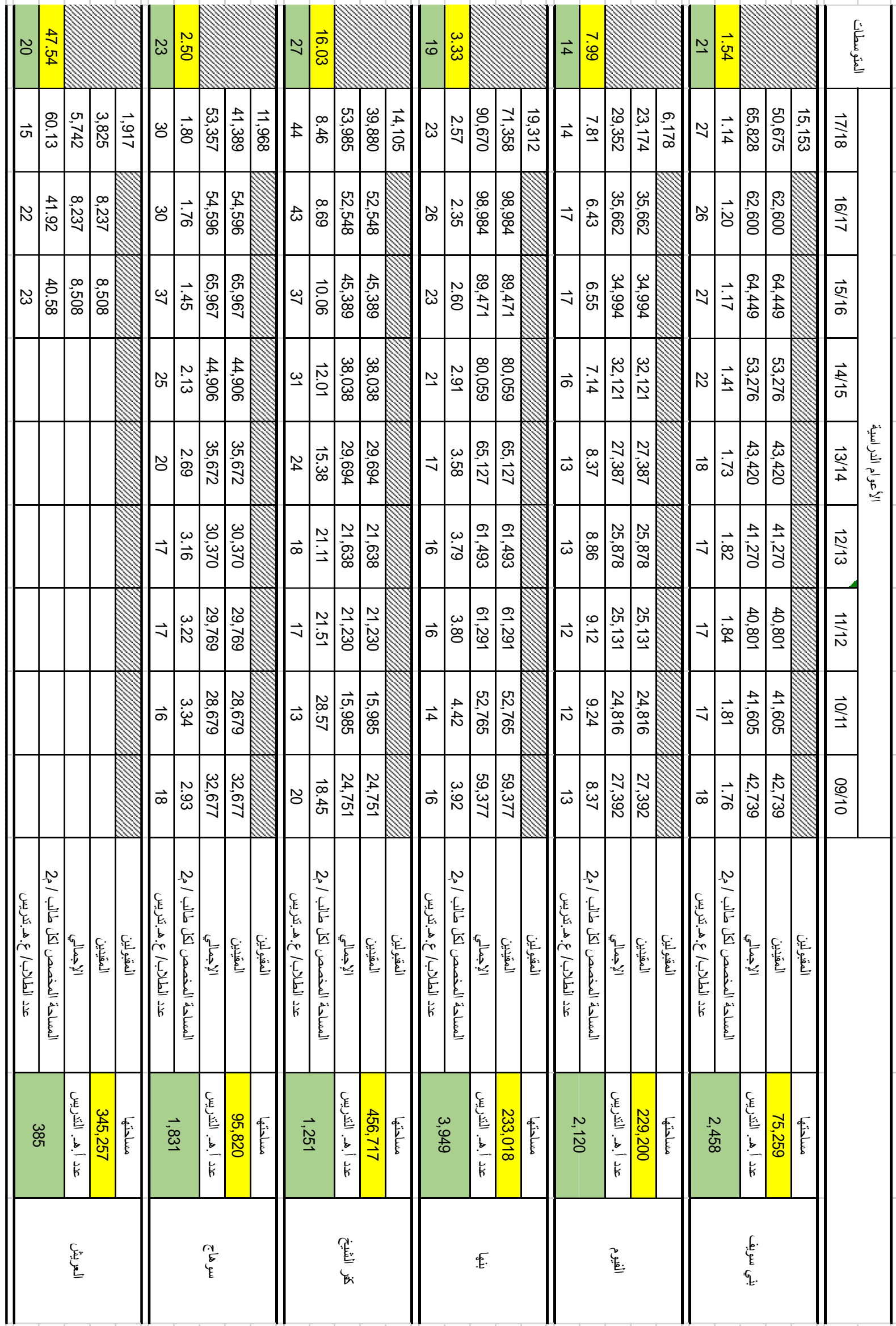


الجامعات الحكومية المصرية "در اسة استقر ائية في نشأة وو اقع الجامعات الحكومية المصرية"

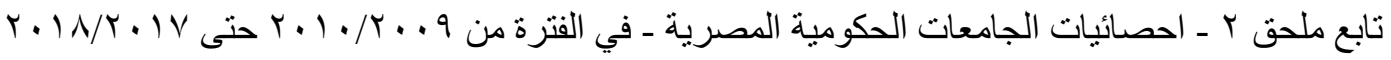

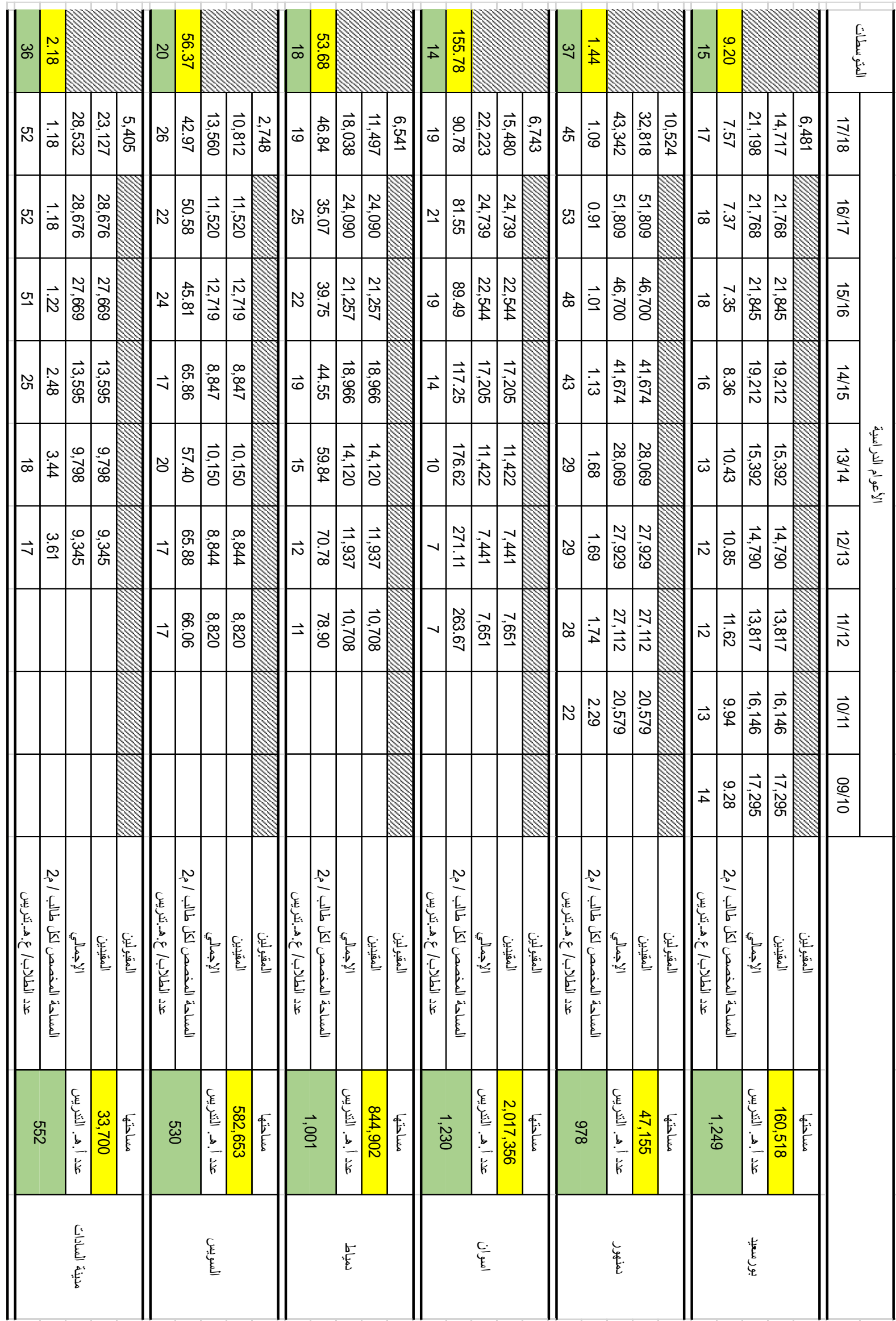

